\title{
Two-dimensional vortex-induced vibration suppression through the cylinder transverse linear/nonlinear velocity feedback
}

Received: 14 February 2017 / Revised: 7 July 2017 / Published online: 18 August 2017

(C) The Author(s) 2017. This article is an open access publication

\begin{abstract}
Suppressing vortex-induced vibration (VIV) has recently attracted numerous researchers due to its practical significance in many engineering applications. Most of the previous studies have focused on a passive or active flow control. A structurally active control approach to mitigate a two-dimensional, nonlinear coupled, cross-flow/in-line VIV has not been well studied. This paper presents a reduced-order fluid-structure dynamic model and combined analytical-numerical solutions for the efficient suppression of two-dimensional VIV of a flexibly mounted circular cylinder in uniform flows. The theoretical model is based on the use of coupled Duffing-Rayleigh oscillators with three variables describing the cylinder cross-flow/in-line displacements and the strength of the fluid vortex circulation in the cylinder wake. These equations of fluid-structure motions contain geometric and hydrodynamic nonlinearities. Closed-loop linear and nonlinear velocity feedback controllers are implemented in the transverse direction governing the larger cross-flow response than the associated in-line counterpart. Approximated analytical expressions are derived by using the harmonic balance to explicitly capture the system nonlinear dynamic features and the effects of key dimensionless parameters. Parametric investigations are carried out to evaluate the linear versus nonlinear controller performance in terms of the maximum response suppression capability and the power requirement in a wide range of reduced flow velocities, mass ratios, and control gains. Over the main lock-in resonance region with coupled cross-flow/inline responses, the linear controller is found to be more efficient in suppressing the two-dimensional VIV by also modifying the system frequencies and phase relationships. Nevertheless, based on the power comparison, the nonlinear control is superior to the linear control for very small targeted controlled amplitudes of a very low-mass cylinder. These active control strategies may be further applied to the multimode VIV suppression of a long flexible cylinder with multi degrees of freedom.
\end{abstract}

\section{Nomenclature}

$\begin{array}{ll}A_{x} / D, A_{y} / D & \text { Cylinder amplitude per diameter } \\ a_{1}-a_{10} & \text { Analytical solution coefficients } \\ C_{D 0}\left(C_{L 0}\right) & \text { Unsteady drag (lift) coefficient of stationary cylinder } \\ c_{S} & \text { Structural viscous damping } \\ c_{f} & \text { Fluid-added damping } \\ C_{a} & \text { Added mass coefficient } \\ D & \text { Cylinder external diameter } \\ D_{t} & \text { Mean drift effect caused by geometric nonlinear coupling }\end{array}$

B. Ma $\cdot$ N. Srinil $(\varangle)$

School of Engineering, Newcastle University, Newcastle upon Tyne, UK

E-mail: narakorn.srinil@newcastle.ac.uk

Tel.: +441912086499

Fax: +44 1912085491 


\begin{tabular}{|c|c|}
\hline$F_{x}^{*}, F_{y}^{*}$ & Dimensional fluid force \\
\hline$F_{y c}$ & Dimensionless control force \\
\hline$F_{y c}^{*}$ & Dimensional control force \\
\hline$f_{o}$ & Cylinder oscillation frequency \\
\hline$f_{n}$ & Cylinder natural frequency \\
\hline$G$ & Dimensional nonlinear gain \\
\hline$Q$ & Dimensional linear gain \\
\hline$K$ & Cylinder stiffness \\
\hline$m_{s}$ & Cylinder mass \\
\hline$m$ & System total mass \\
\hline$m^{*}$ & Mass ratio \\
\hline$m_{a}$ & Fluid-added mass \\
\hline$p$ & Wake variable transformation \\
\hline$P$ & Dimensionless average power \\
\hline$q\left(q_{0}\right)$ & Dimensionless displacement (amplitude) of vortex circulation \\
\hline $\mathrm{Re}$ & Reynolds number \\
\hline$R$ & Maximum amplitude reduction percentage \\
\hline St & Strouhal number \\
\hline$t$ & Dimensional time \\
\hline$U$ & Dimensional flow velocity \\
\hline$V_{r}$ & Reduced flow velocity \\
\hline$X(x)$ & Dimensional (dimensionless) in-line displacement \\
\hline$Y(y)$ & Dimensional (dimensionless) cross-flow displacement \\
\hline$x_{0}, y_{0}$ & Dimensionless amplitude \\
\hline$q_{M}, x_{M}, y_{M}$ & Dimensionless amplitude at ideal lock-in frequency $(\delta=\omega=1)$ \\
\hline$\alpha_{x}^{*} \beta_{x}^{*} \alpha_{y}^{*} \beta_{y}^{*}$ & Dimensional geometrically nonlinear coefficients \\
\hline$\alpha_{x} \beta_{x} \alpha_{y} \beta_{y}$ & Dimensionless geometrically nonlinear coefficients \\
\hline$\alpha_{x q}, \alpha_{y q}$ & Hydrodynamic force coefficients \\
\hline$\beta_{K}$ & Dimensionless linear control gain \\
\hline$\beta, \varepsilon, \lambda$ & Empirical wake coefficients \\
\hline$\gamma$ & Stall parameter \\
\hline$\gamma_{\mathrm{G}}$ & Dimensionless nonlinear control gain \\
\hline$\delta$ & Cylinder-to-vortex-shedding frequency ratio \\
\hline$\theta_{x y}$ & $x-y$ relative phase angle \\
\hline$\theta_{q y}$ & $q-y$ relative phase angle \\
\hline$\mu$ & Dimensionless mass parameter \\
\hline$\xi$ & Structural damping ratio \\
\hline$\rho$ & Fluid density \\
\hline$\tau$ & Dimensionless time \\
\hline$\omega$ & Dimensionless oscillation frequency in analytical solution \\
\hline$\omega_{n}$ & Cylinder angular natural frequency \\
\hline$\omega_{s t}$ & Vortex-shedding angular frequency \\
\hline
\end{tabular}

\section{Introduction}

Offshore cylindrical structures such as spar platforms, subsea risers, cables, mooring lines, flowlines, pipelines, jumpers, and vertical tensioned legs are widely used in the oil and gas industry. To ensure their engineering efficiency, integrity, and safety throughout the lifespans, the nonlinear fluid-structure interaction effects associated with the resonant vortex-induced vibration (VIV) must be controlled. Alongside theoretical and experimental studies which have revealed a variety of key multi-physics VIV phenomena [5,32,39], the subject of VIV control has also drawn a considerable attention over the past decades. Several passive/active flow control strategies have been developed and verified to justify their vibration control performances versus potential installation, operational and maintenance costs. In particular, passive flow control devices such as strakes and fairings have been widely used by the oil and gas industrialists for riser VIV control $[9,29,31,40]$. However, it is well known that the introduction of such devices along the long slender structure modifies the structural 
configuration and increases the drag forces, apart from their high costs in manufacturing, installation, and maintenance. Alternatively, active flow controls by introducing, for instance, a surface suction and blowing [8], an acoustic actuation [15,21], and a neighboring rotational cylinder [14,20,24] have also been explored with the aim of delaying, interrupting, or intervening in the vortex formation process in the wake. Nevertheless, due to the frequent changes and uncertainties in ocean environments, it might be impractical to accurately predict the space-time-varying vortex formations and implement the active flow control methods in real time for deep-water structures. To overcome these challenges, the structurally active controls by implementing sensors and actuators at a critical location along the structure span and by enhancing the system damping performance could provide alternative solutions for efficient VIV suppression [3].

For the structurally active control of cross-flow-only VIV with a single degree of freedom (DOF), Baz and Ro [4] experimentally investigated the influence of a velocity feedback controller which can generate an adversely proportional signal to the cylinder velocity. The control action on the cylinder was achieved by an electromagnetic actuator which attenuated VIV response by more than $80 \%$ in the main synchronization region. Their experimental results were also validated by a theoretical model based on the use of a van der Pol-type wake oscillator. Such a wake oscillator model has recently been refined by Facchinetti et al. [13]. By tuning of empirical parameters to match experimental observations, theoretical predictions were found to show a good agreement with controlled and uncontrolled experimental results. A velocity feedback control for a circular cylinder with a very high mass ratio was studied by Mehmood et al. [27] where linear/nonlinear active feedback controllers for cross-flow VIV were numerically investigated using the computational fluid dynamics (CFD) approach in a very low Reynolds number (Re) range of 95-125. Both controllers were found to be functional in reducing VIV within the lock-in range. The optimal law for the desired controlled amplitudes was studied through the power requirement comparisons which revealed the control efficiency depending on the targeted reduced amplitudes. In addition to experimental and CFD studies, the VIV controller analysis was recently treated by Dai et al. [12] using a wake oscillator model and accounting for the effects of control gain and time-delayed term. By properly adjusting the controller parameters, effective VIV reduction could be achieved. Nevertheless, toward realistic applications, the effect of in-line VIV and combined cross-flow/in-line VIV suppression should be considered.

As regards the 2-DOF VIV, investigation into the VIV active controller for the strongly coupled fluidstructure interactions is still very limited owing to a lack of theoretical prediction models which have just recently been developed and validated $[1,37]$. Several experimental $[11,23,38]$ and CFD $[2,30]$ 2-DOF VIV studies have enabled the recently modified wake oscillator models to capture the experimentally observed hydro-elastic phenomena governing coupled cross-flow/in-line VIV alongside the lift/drag hydrodynamic features [41]. By using a CFD approach, Hasheminejad et al. [19] recently proposed an adaptive fuzzy sliding mode controller for suppressing the 2-DOF VIV for a low-mass cylinder in a laminar flow with $\mathrm{Re}=90$. The cross-flow control force was considered, and the in-line motion was suppressed due to the coupling of cross-flow/in-line VIV, even at such a low Re. Nevertheless, practical subcritical flow applications in a higher Re range and understanding of various aspects related to the 2-DOF VIV active controls including the effects of control gain, cylinder mass-damping ratio and reduced flow velocity, are still needed. These aspects will be the research focus of this study.

This paper presents theoretical models and combined analytical-numerical approaches to investigate the structurally active linear and nonlinear controls for effectively suppressing the two-dimensional coupled cross-flow/in-line VIV of circular cylinders. The paper is structured as follows. The mathematical model for the structurally active controls of the 2-DOF cylinder VIV is presented in Sect. 2. By using the harmonic balance method in Sect. 3, the analytical solution of the governing nonlinear equations is derived to establish some closed-form expressions and parametric relationships of the dynamic system in the presence of control. In Sect. 4, the theoretical model is calibrated, and the parametric studies are carried out to highlight several aspects related to linear/nonlinear active controls. The paper ends with the conclusions in Sect. 5.

\section{Nonlinear fluid-structure dynamic model}

A structurally active controller can be represented by a mechanical device (e.g., an electromagnetic actuator) installed inside the cylinder to avoid disturbing the external flow fields [4]. This concept is intrinsically different from the active flow control strategy whose aim is to disrupt or interfere with the vortex formations. Because of the two-dimensionally nonlinear coupling of cross-flow $(Y)$ and in-line $(X)$ motions, the actuator 
can be activated in either $Y$ or $X$ direction with a control gain function imparting an adaptive damping force proportional to the cylinder velocity. This improvement of damping performance by the time-varying feedback control force proves to be the most reliable scheme for the active control [22].

Attention is placed on the 2-DOF VIV suppression in the main critical lock-in region. The transverse $Y$ controller is implemented due to its larger response than the associated in-line motion. The $X$ controller can be considered in the pure in-line VIV range at lower reduced velocities. Both linear control (LC) and nonlinear control (NC) are employed and compared in order to justify (i) whether the former is sufficient for suppressing the VIV fluid-structure interactions with system nonlinearities, (ii) whether the latter should be accounted for due to potential higher-order nonlinear effects, and (iii) whether both strategies can be efficiently used for the coupled 2-DOF VIV mitigation of circular cylinders with different mass ratios and power requirements.

Figure 1a displays a schematic idealization of a flexibly mounted rigid circular cylinder placed in uniform flow of velocity $U$. The cylinder consists of a spring-mass-damping and $Y$ controller with two degrees of freedom (DOF) of oscillations. A block diagram of the active control strategy is also displayed in Fig. 1b. The nonlinear ordinary differential equations of coupled $X-Y$ cylinder motions, which are subjected to in-line $\left(F_{x}^{*}\right)$ and cross-flow $\left(F_{y}^{*}\right)$ VIV excitations and control $\left(F_{y c}^{*}\right)$ force per unit length, may be expressed as

$$
\begin{aligned}
\left(m_{s}+m_{a}\right) \ddot{X}+\left(c_{s}+c_{f}\right) \dot{X}+K\left(X+\alpha_{x}^{*} X^{3}+\beta_{x}^{*} X Y^{2}\right) & =F_{x}^{*}, \\
\left(m_{s}+m_{a}\right) \ddot{Y}+\left(c_{s}+c_{f}\right) \dot{Y}+K\left(Y+\alpha_{y}^{*} Y^{3}+\beta_{y}^{*} Y X^{2}\right) & =F_{y}^{*}-F_{y c}^{*},
\end{aligned}
$$

where an overdot denotes differentiation with respect to the dimensional time $t . m_{s}$ is the structural mass, $m_{a}$ the fluid-added mass, $m_{a}=C_{a} \rho \pi D^{2} / 4$, with $C_{a}$ being the added mass coefficient assumed to be unity for a circular cylinder [33], $\rho$ the fluid density, $D$ the cylinder diameter, $K$ the linear elastic stiffness coefficient, $c_{s}$ and $c_{f}$ the structural viscous and fluid-added damping coefficient, respectively.

It is worth noting that in practice $K, c_{s}$ and $c_{f}$ may be dissimilar between $X$ and $Y$ directions [38]; however, they are, herein, assumed to be equal in both directions to maintain the symmetry of cylinder properties. Following Facchinetti et al. [13], $c_{f}$ may be fixed and defined as $c_{f}=\gamma \omega_{n} \rho D^{2}$ in which $\omega_{n}$ is the angular natural frequency of the cylinder in still water and $\gamma$ is the stall parameter [34] providing a self-limiting response in the absence of $c_{s}$. Note also that, from a phenomenological modeling viewpoint, $m_{a}$ and $c_{f}$ in Eqs. (1) and (2) are assumed to be associated with the oscillating cylinder in still water, whereas their nonlinear dynamic counterparts subject to VIV are captured through the wake oscillator model [41]. Separating $m_{a}$ and $c_{f}$ from the total force expressions allows one to normalize Eqs. (1) and (2) into general dimensionless forms. The geometrically nonlinear stiffness terms with the associated parameters $\left(\alpha_{x}^{*}, \beta_{x}^{*}, \alpha_{y}^{*}, \beta_{y}^{*}\right)$ govern the physical stretching and the two-dimensional displacement coupling observed experimentally [38]. These cubic-type Duffing terms [25] are accounted for since they enable the model to capture the key hysteresis effect with a response jump in the large-amplitude dual-resonant VIV for a low-mass cylinder [37].

The LC force can be expressed as $F_{y c}^{*}=Q \dot{Y}$ whereas the NC one reads $F_{y c}^{*}=G \dot{Y}^{3}$, with a positive control gain $(Q, G)$ to be assigned and varied [28]. The cubic-type NC is considered since the cylinder nonlinearities (Eqs. 1, 2) and the Rayleigh wake oscillator (Eq. 4) are theoretically of cubic type. As for the hydrodynamic excitation forces associated with the two-DOF VIV of a rigid cylinder, there are a few phenomenological models available in the literature. Herein, the fluid physics-based model-which has been derived from the vortex strength principle and requires a single variable describing the fluid displacement circulation $q$-is considered. In-depth details can be found in Bai and Qin [1] where the time-varying in-line and cross-flow fluid forces read $F_{x}^{*}(t)=-\rho C_{D 0} D^{4} \dot{q} \ddot{q} /\left(32 \pi^{3} S t^{3} U\right)$ and $F_{y}^{*}(t)=C_{L 0} \rho U D^{2} \dot{q} /(8 \pi S t)$. These force functions contain empirical quantities, namely the Strouhal number $(\mathrm{St})$, the unsteady lift $\left(C_{L 0}\right)$ and $\operatorname{drag}\left(C_{D 0}\right)$ force coefficients of the stationary cylinder. Note that the effect of static mean drag force and its amplification due to VIV [42] is herein neglected since attention is placed on the active control of the system fluctuating dynamics, rather than the statics.

By introducing the dimensionless time $\tau=t \omega_{s t}$ in which $\omega_{s t}$ is the angular vortex-shedding frequency, and the normalized displacements $x=X / D$ and $y=Y / D$, the nonlinearly coupled equations of cylinder in-line and cross-flow motions can be expressed, in dimensionless form, as

$$
\begin{aligned}
& \ddot{x}+(2 \xi \delta+\gamma / \mu) \dot{x}+\delta^{2}\left(x+\alpha_{x} x^{3}+\beta_{x} x y^{2}\right)=-2 \alpha_{x q} \dot{q} \ddot{q}, \\
& \ddot{y}+(2 \xi \delta+\gamma / \mu) \dot{y}+\delta^{2}\left(y+\alpha_{y} y^{3}+\beta_{y} y x^{2}\right)=\alpha_{y q} \dot{q}-F_{y c},
\end{aligned}
$$

in which an overdot now denotes differentiation with respect to the dimensionless time $\tau$. For LC (NC), $F_{y c}=\beta_{K} \dot{y}\left(F_{y c}=\gamma_{G} \dot{y}^{3}\right)$. Dimensionless parameters include the damping ratio $\xi=c_{s} /\left(2 m \omega_{n}\right)$, frequency 
(a)

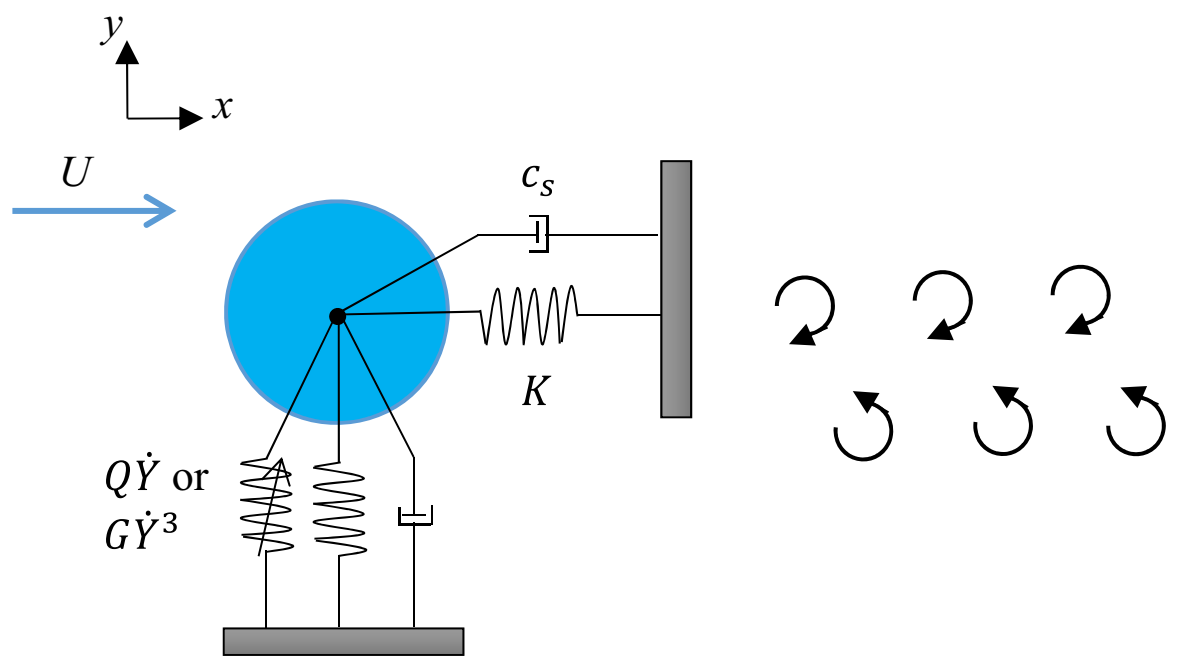

(b)

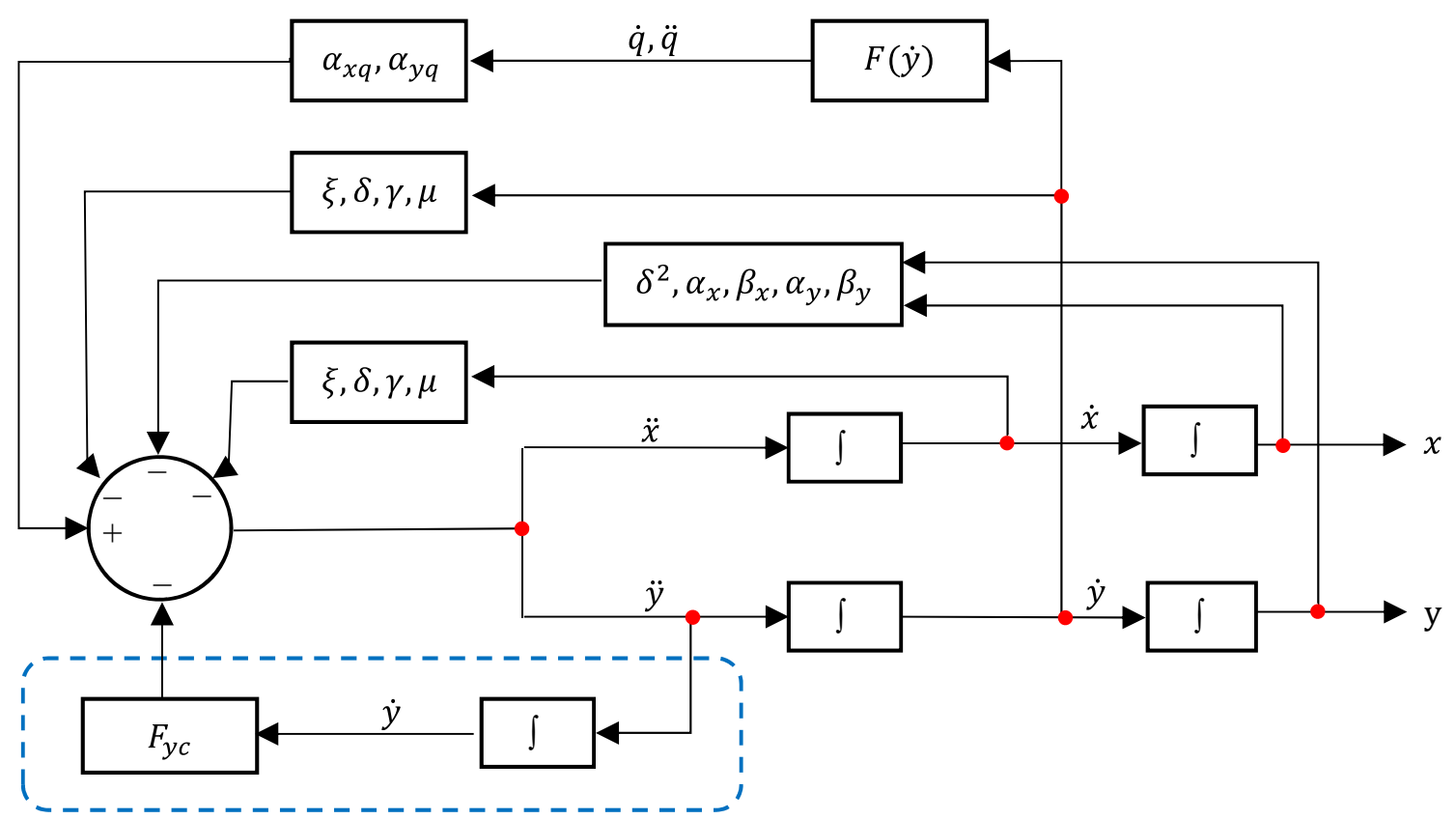

Control Loop

Fig. 1 An active velocity feedback control model of an elastically mounted circular cylinder undergoing two-dimensional VIV (a) and a block diagram of active control strategy (b)

ratio $\delta=\omega_{n} / \omega_{s t}$, geometric coefficients $\left(\alpha_{x}, \beta_{x}, \alpha_{y}, \beta_{y}\right)$, force coefficients $\alpha_{x q}=C_{D 0} /\left(32 \pi^{2} \mathrm{St}^{2} \mu\right)$ and $\alpha_{y q}=C_{L 0} /\left(16 \pi^{2} \mathrm{St}^{2} \mu\right)$, control gain $\beta_{K}=Q / m \omega_{s t}$ and $\gamma_{G}=G D^{2} \omega_{s t} / m$, with $\mu=m / \rho D^{2}$ and $m=$ $m_{s}+m_{a}$. The nominal reduced velocity parameter $V_{r}$ can be related to $\delta$ through $\delta=1 /\left(\mathrm{St} V_{r}\right)$ since $V_{r}=$ $2 \pi U / \omega_{n} D$. For parametric studies in Sect. $4, V_{r}$ is varied through $\delta$ in Eqs. (3) and (4). It is important to note that the quadratic nonlinear coupling term as a function of fluid $\dot{q} \ddot{q}$ appears in Eq. (3) whereas the typical linear coupling term $\dot{q}$ appears in Eq. (4). Such quadratic term in the in-line equation is responsible for the appearance of a figure-of-eight $X-Y$ trajectory [37] associated with a dual 2:1 resonance $[10,38]$. To describe the fluid displacement circulation $q$, a single Rayleigh [18] wake oscillator may be written as [1] 


$$
\ddot{q}-\varepsilon\left(1-\lambda \dot{q}^{2}\right) \dot{q}+q=\beta \dot{y},
$$

in which $\varepsilon, \beta$, and $\lambda$ are the system empirical wake coefficients which can be specified or tuned by calibrating with experimental data; see Sect. 4.1. They can also be functions of system properties such as the mass ratio $\left(m^{*}=m /\left(\rho \pi D^{2} / 4\right)\right)[35,38]$ and Re [36]. The form in Eq. (5) with the cylinder velocity coupling term $\beta \dot{y}$ is different from the van der Pol equation [16] typically employed in the literature with the wake damping $\varepsilon\left(1-q^{2}\right) \dot{q}$ and acceleration coupling $\beta \ddot{y}$ terms [13]. Nevertheless, through a variable transformation by letting $\dot{q}=p$, the van der Pol oscillator of $p$ can be rewritten [28]. For efficient numerical computations and convenience in the analytical formulation, only Eq. (5) is considered for the 2-DOF VIV, instead of using double van der Pol oscillators previously considered by Srinil and Zanganeh [37].

Overall, the nonlinearly coupled equations (3-5) contain several empirical parameters. Through a number of parametric and sensitivity studies whose selected results will be presented in Sects. 4 and 5, we assign $\lambda=0.2, \gamma=0.5, \mathrm{St}=0.19, C_{D 0}=0.2$ and $C_{L 0}=0.3$, as in Bai and Qin [1], and $\alpha_{x}=\beta_{x}=\alpha_{y}=\beta_{y}=0.4$. Some of these parameters may be treated as random variables [26] due to variations in different sets of experimental data. Some values (St, $\left.C_{D 0}, C_{L 0}\right)$ are suitable for a cylinder with a smooth surface and subject to a subcritical flow with $\operatorname{Re}<2 \times 10^{5}$ [6]. Empirical wake oscillator coefficients $(\varepsilon, \beta)$ will be deduced in Sect. 4.1. For numerical integrations, a fourth-order Runge-Kutta scheme can be used with initial conditions of $x=y=\dot{x}=\dot{y}=\dot{q}=0$ and $q=2$ and with a fixed dimensionless time step of 0.01 providing a convergence of steady-state simulation results. Note that the present model has not yet accounted for the Re dependence of response amplitudes as underlined by [17]. With new and substantial experimental data in a wide range of system parameters for the 2-DOF VIV, the Re effect could be further incorporated into the model, e.g., through the empirical wake coefficients which regulate the self-limiting $(\varepsilon)$ and fluid-cylinder coupling $(\beta)$ terms in Eq. (5). The Re dependence of wake coefficients has been demonstrated by Srinil [35] for the cross-flow VIV prediction of long flexible cylinders.

\section{Analytical prediction of controlled responses}

To gain insights into the two-dimensionally controlled VIV responses and explicitly capture the nonlinear coupling of key physical parameters, analytical expressions are derived which can complement numerical integration results. To capture the most influential effects of system nonlinearities, the first-order harmonic balance approach is applied. Due to the natural occurrence of a periodic 2:1 resonance of the cylinder $X-Y$ response in a wide range of $V_{r}[11,38]$, it is reasonable to assume a periodic solution of cross-flow response $(y)$ and vortex wake circulation $(q)$ with a dimensionless resonant oscillation frequency $(\omega)$ as in Facchinetti et al. [13], whereas the cylinder in-line motion $(x)$ can be treated as a harmonic motion at $2 \omega$. Accordingly, the approximated motions for $x, y$ and $q$ may be expressed as

$$
\begin{aligned}
& x=x_{0} \sin \left(2 \omega \tau+\theta_{x y}\right), \\
& y=y_{0} \sin (\omega \tau), \\
& q=q_{0} \sin \left(\omega \tau+\theta_{q y}\right),
\end{aligned}
$$

in which $x_{0}, y_{0}$, and $q_{0}$ are the dimensionless oscillation amplitudes and $\theta_{x y}\left(\theta_{q y}\right)$ is the associated $x-y(q-y)$ phase relationship. The LC system is first considered. By substituting Eqs. (6-8) into Eqs.(3-5) with $F_{y c}=$ $\beta_{K} \dot{y}$, expanding and balancing the trigonometric terms in the forms of $\sin \left(2 \omega t+\theta_{x y}\right), \cos \left(2 \omega t+\theta_{x y}\right), \sin (\omega t)$, $\cos (\omega t), \sin \left(\omega t+\theta_{q y}\right)$ and $\cos \left(\omega t+\theta_{q y}\right)$, and neglecting the higher harmonic terms, the associated expressions can be obtained, respectively, as

$$
\begin{gathered}
-4 \omega^{2} x_{0}+\delta^{2}\left(x_{0}+\alpha_{x} \frac{3 x_{0}^{3}}{4}+\beta_{x} \frac{x_{0} y_{0}^{2}}{2}\right)=\alpha_{x q} q_{0}^{2} \omega^{3} \cos \left(2 \theta_{q y}-\theta_{x y}\right), \\
2\left(2 \xi \delta+\frac{\gamma}{\mu}\right) \omega x_{0}=\alpha_{x q} q_{0}^{2} \omega^{3} \sin \left(2 \theta_{q y}-\theta_{x y}\right) \\
-\omega^{2} y_{0}+\delta^{2}\left(y_{0}+\alpha_{y} \frac{3 y_{0}^{3}}{4}+\beta_{y} \frac{x_{0}^{2} y_{0}}{2}\right)=-\alpha_{y q} \omega q_{0} \sin \left(\theta_{q y}\right) \\
\left(2 \xi \delta+\frac{\gamma}{\mu}\right) \omega y_{0}+\left(\beta_{K} \omega y_{0}\right)=\alpha_{y q} \omega q_{0} \cos \left(\theta_{q y}\right)
\end{gathered}
$$




$$
\begin{gathered}
q_{0}\left(1-\omega^{2}\right)=\beta \omega y_{0} \sin \left(\theta_{q y}\right), \\
\frac{3}{4} \varepsilon \lambda q_{0}^{3} \omega^{3}-\varepsilon \omega q_{0}=\beta \omega y_{0} \cos \left(\theta_{q y}\right) .
\end{gathered}
$$

The pairs of Eqs. (9) and (10), Eqs. (11) and (12), and Eqs. (13) and (14) are derived from Eq. (3), (4) and (5), respectively. By dividing Eq. (10) by Eq. (9) and summing the squares of them, Eqs. (15) and (16) are obtained, respectively. Similarly, the combination of Eqs. (11) and (12) gives rise to the first expression in Eq. (17) and to Eq. (18), whereas the combination of Eqs. (13) and (14) entails the second expression in Eq.(17) and Eq. (19), respectively. These are written as follows.

$$
\begin{aligned}
\tan \left(2 \theta_{q y}-\theta_{x y}\right) & =\frac{2(2 \xi \delta+\gamma / \mu) \omega}{-4 \omega^{2}+\delta^{2}\left(1+\alpha_{x} \frac{3 x_{0}^{2}}{4}+\beta_{x} \frac{y_{0}^{2}}{2}\right)}, \\
\left(\alpha_{x q} q_{0}^{2} \omega^{3}\right)^{2} & =\left[2\left(2 \xi \delta+\frac{\gamma}{\mu}\right) \omega x_{0}\right]^{2}+\left[-4 \omega^{2} x_{0}+\delta^{2}\left(x_{0}+\alpha_{x} \frac{3 x_{0}^{3}}{4}+\beta_{x} \frac{x_{0} y_{0}^{2}}{2}\right)\right]^{2}, \\
\tan \left(\theta_{q y}\right) & =\frac{\omega^{2}-\delta^{2}\left(1+\alpha_{y} \frac{3 y_{0}^{2}}{4}+\beta_{y} \frac{x_{0}^{2}}{2}\right)}{\left(2 \xi \delta+\frac{\gamma}{\mu}\right) \omega+\left(\beta_{K} \omega\right)}=\frac{\left(1-\omega^{2}\right)}{\frac{3}{4} \varepsilon \lambda q_{0}^{2} \omega^{3}-\varepsilon \omega}, \\
\left(\alpha_{y q} \omega q_{0}\right)^{2} & =\left[\left(2 \xi \delta+\frac{\gamma}{\mu}\right) \omega y_{0}+\left(\beta_{K} \omega y_{0}\right)\right]^{2}+\left[-\omega^{2} y_{0}+\delta^{2}\left(y_{0}+\alpha_{y} \frac{3 y_{0}^{3}}{4}+\beta_{y} \frac{x_{0}^{2} y_{0}}{2}\right)\right]^{2}, \\
\frac{y_{0}}{q_{0}} & =\frac{\sqrt{\left(1-\omega^{2}\right)^{2}+\left(\varepsilon \omega-\frac{3}{4} \varepsilon \lambda q_{0}^{2} \omega^{3}\right)^{2}}}{\beta \omega} .
\end{aligned}
$$

Due to the nonlinear $X-Y$ displacement coupling term $\left(x y^{2}\right)$ in Eq. (3), the associated mean $\operatorname{drift}$ effect $\left(D_{t}\right)$ is generated which can be expressed in dimensionless form as

$$
D_{t}=\frac{-\delta^{2} \beta_{x} x_{0} y_{0}^{2} \sin \left(\theta_{x y}\right)}{4} .
$$

This drift effect is dependent on the cylinder properties including amplitudes $\left(x_{0}, y_{0}\right)$, frequency ratio $(\delta)$, stiffness $\left(\beta_{x}\right)$, and phase difference $\left(\theta_{x y}\right)$ associated with the figure-of-eight orbital $X-Y$ motion. From Eq. (20), the zero or maximum $\left|D_{t}\right|$ occurs when $\theta_{x y}=n \pi(n=0,1,2 \ldots)$ or $(n+1) \pi / 2(n=0,2, \ldots)$, respectively, with a negative (positive) $D_{t}$ suggesting an in-line downstream (upstream) drift. This $D_{t}$ value should be recognized when performing numerical simulations and experiments [38].

Equations (17) and (18) are further rearranged as

$$
\begin{gathered}
\frac{\left(2 \xi \delta+\frac{\gamma}{\mu}+\beta_{K}\right)\left(1-\omega^{2}\right)}{\varepsilon-\frac{3}{4} \varepsilon \lambda q_{0}^{2} \omega^{2}}=-\omega^{2}+\delta^{2}\left(1+\alpha_{y} \frac{3 y_{0}^{2}}{4}+\beta_{y} \frac{x_{0}^{2}}{2}\right), \\
\left(\alpha_{y q} \omega \frac{q_{0}}{y_{0}}\right)^{2}=\left[\left(2 \xi \delta+\frac{\gamma}{\mu}\right) \omega+\left(\beta_{K} \omega\right)\right]^{2}+\left[-\omega^{2}+\delta^{2}\left(1+\alpha_{y} \frac{3 y_{0}^{2}}{4}+\beta_{y} \frac{x_{0}^{2}}{2}\right)\right]^{2} .
\end{gathered}
$$

Then, by combining Eqs. (21) and (22), we obtain

$$
\left(\alpha_{y q} \omega \frac{q_{0}}{y_{0}}\right)^{2}=\left[\left(2 \xi \delta+\frac{\gamma}{\mu}\right) \omega+\left(\beta_{K} \omega\right)\right]^{2}+\left[\frac{\left(2 \xi \delta+\frac{\gamma}{\mu}+\beta_{K}\right)\left(1-\omega^{2}\right)}{\varepsilon-\frac{3}{4} \varepsilon \lambda q_{0}^{2} \omega^{2}}\right]^{2}
$$


By substituting Eq. (19) for $q_{0} / y_{0}$ into Eq. (23), and analytically solving the resulting equation, the closed-form expression for the two possible values of $q_{0}$ reads

$$
\begin{aligned}
& q_{0}=\sqrt{\frac{4 \varepsilon \omega+2\left(1 \pm \sqrt{1-4 \mathrm{a}_{1}^{2} \mathrm{a}_{2}^{2}}\right) / a_{2}}{3 \varepsilon \lambda \omega^{3}},} \\
& a_{1}=1-\omega^{2}, \quad a_{2}=\frac{2 \xi \delta+\gamma / \mu+\beta_{K}}{\alpha_{y q} \beta \omega} .
\end{aligned}
$$

It can be appreciated that the vortex force amplitude $q_{0}$ is controlled through $a_{2}$ with a series of damping terms. Once a positive real value of $q_{0}$ is obtained from Eq. (24), that of $y_{0}$ can be determined via Eq. (19). To derive a closed-form expression for $x_{0}$, Eq. (16) is further rearranged as a sixth-order polynomial equation governing $x_{0}$. From numerical simulation checks, it is found that the $x_{0}^{6}$ term is negligible $(<0.02)$ since generally $x_{0}<0.5$; hence, by considering the resulting equation accounting for the next highest order of $x_{0}^{4}$, the unique solution for $x_{0}$ can be derived as

$$
\begin{aligned}
& x_{0}=\sqrt{\frac{2 \sqrt{\frac{a_{3}^{2}}{4}+\frac{a_{3} a_{4}^{2}}{2}+\frac{a_{4}^{4}}{4}+2 a_{6} a_{5} a_{4}}-a_{4}^{2}-a_{3}}{4 a_{4} a_{5}}}, \\
& a_{3}=4 \omega^{2}\left(2 \xi \delta+\frac{\gamma}{\mu}\right)^{2}, \quad a_{4}=-4 \omega^{2}+\delta^{2}+\beta_{x} \frac{y_{0}^{2} \delta^{2}}{2}, \quad a_{5}=\alpha_{x} \frac{3 \delta^{2}}{4}, \quad a_{6}=\left(\alpha_{x q} q_{0}^{2} \omega^{3}\right)^{2} .
\end{aligned}
$$

Depending on the system parameters and empirical coefficients, it can be appreciated that both $y_{0}$ (Eq. 19) and $x_{0}$ (Eq. 26) are nonlinear functions of $q_{0}$, and the reduction of $x_{0}$ is dependent on the suppressed $y_{0}$ due to their nonlinear coupling. Subsequently, the system phase differences $\theta_{q y}$ and $\theta_{x y}$ can be obtained through Eqs. (17) and (15), respectively.

For NC system, by substituting Eqs. (6-8) into Eqs. (3-5) with $F_{y c}=\gamma_{G} \dot{y}^{3}$ and applying the harmonic balance, Eqs. (9-22) from the LC system can be used with $\gamma_{G}\left(3 \omega^{3} y_{0}^{3}\right) / 4$ replacing $\beta_{K} \omega y_{0}$ in Eqs. (12) and (18), $\gamma_{G}\left(3 \omega^{3} y_{0}^{2}\right) / 4$ replacing $\beta_{K} \omega$ in Eqs. (17), (22) and (23), and $\gamma_{G}\left(3 \omega^{2} y_{0}^{2}\right) / 4$ replacing $\beta_{K}$ in Eq. (21). By combining the resulting Eqs. (21) and (22) with the above substitutions, the following expression reads

$$
\left(\alpha_{y q} \omega q_{0}\right)^{2}=\left[\left(2 \xi \delta+\frac{\gamma}{\mu}\right) \omega y_{0}+\gamma_{G} \frac{3 \omega^{3} y_{0}^{3}}{4}\right]^{2}+\left\{\frac{\left[\left(2 \xi \delta+\frac{\gamma}{\mu}\right) y_{0}+\gamma_{G} \frac{3 \omega^{2} y_{0}^{3}}{4}\right]\left(1-\omega^{2}\right)}{\varepsilon-\frac{3}{4} \varepsilon \lambda q_{0}^{2} \omega^{2}}\right\}^{2} .
$$

Accordingly, due to the presence of nonlinear amplitudes $\left(y_{0}^{2}, y_{0}^{3}\right)$, it is unfeasible to further derive analytical expressions. Nevertheless, for a specific $V_{r}$ and $\omega$, Eqs. (16), (19) and (28) can be simultaneously solved for the key unknown $q_{0}, y_{0}$ and $x_{0}$. Both $\theta_{q y}$ and $\theta_{x y}$ can then be obtained from Eqs. (17) and (15), respectively.

Next, by imposing the ideal perfect resonance or lock-in condition with $\omega=\delta=1$ for which $V_{r}=1 / \mathrm{St}$ [13], the linearly controlled vortex force and $Y$ response, based on Eqs. (23) and (24), can be predicted, respectively, by

$$
\begin{aligned}
q_{M} & =2 \sqrt{\frac{\left[\varepsilon+\alpha_{y q} \beta \omega /\left(2 \xi+\gamma / \mu+\beta_{K}\right)\right]}{3 \varepsilon \lambda}} \\
y_{M} & =\frac{2 \alpha_{y q}}{\left(2 \xi+\gamma / \mu+\beta_{K}\right)} \sqrt{\left[1+\frac{\beta}{\varepsilon} \frac{\alpha_{y q}}{\left(2 \xi+\gamma / \mu+\beta_{K}\right)}\right] \frac{1}{3 \lambda}} .
\end{aligned}
$$

Correspondingly, with Eqs. (26) and (27), the linearly controlled $X$ response reads

$$
\begin{gathered}
x_{M}=\sqrt{\frac{2 \sqrt{\frac{a_{7}^{2}}{4}+\frac{a_{7} a_{8}^{2}}{2}+\frac{a_{8}^{4}}{4}+2 a_{10} a_{9} a_{8}}-a_{8}^{2}-a_{7}}{4 a_{8} a_{9}},} \\
a_{7}=4\left(2 \xi+\frac{\gamma}{\mu}\right)^{2}, \quad a_{8}=\beta_{x} \frac{y_{M}^{2}}{2}-3, \quad a_{9}=\frac{3}{4} \alpha_{x}, \quad a_{10}=16 \alpha_{x q}^{2}\left[1+\frac{\alpha_{y q} \beta}{\left(2 \xi+\frac{\gamma}{\mu}+\beta_{K}\right) \varepsilon}\right]^{2} .
\end{gathered}
$$



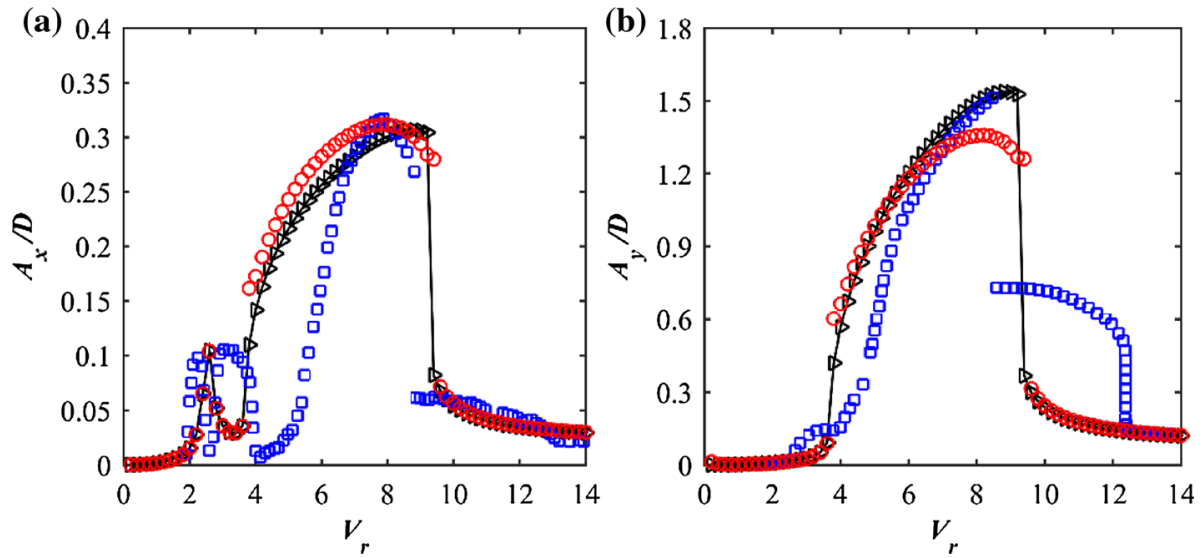

Fig. 2 Comparisons of response amplitudes in the absence of control with numerical (triangles), analytical (circles) and experimental (squares) results for cylinder with $m^{*}=2.6$ and $\xi=0.00361$
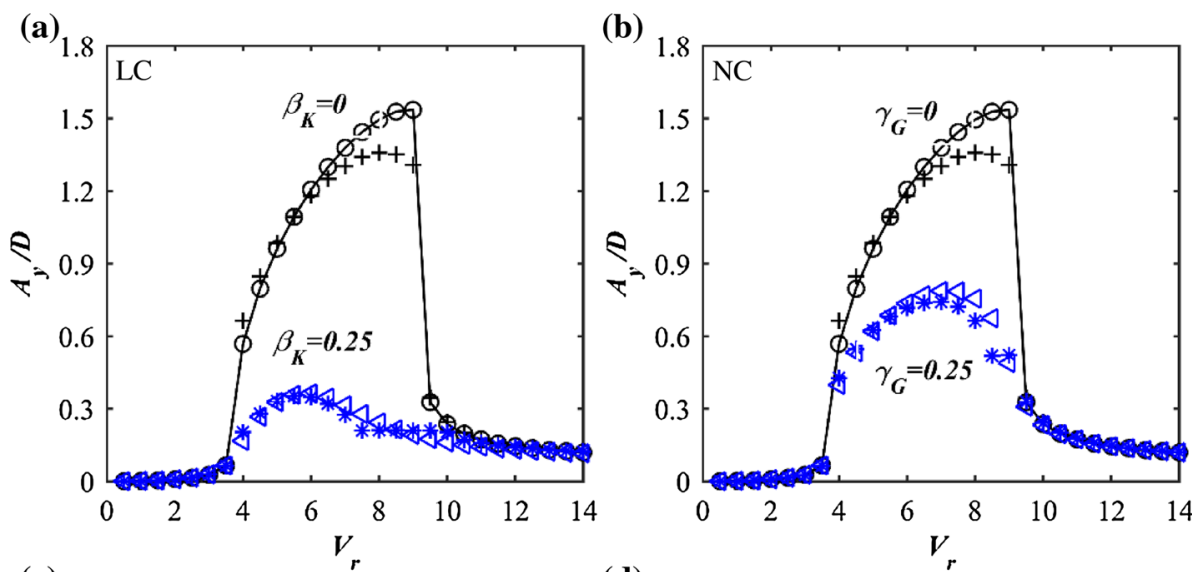

(c)

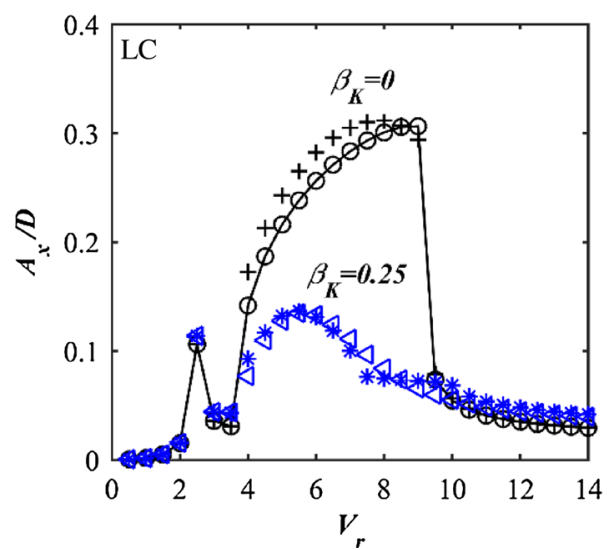

(d)

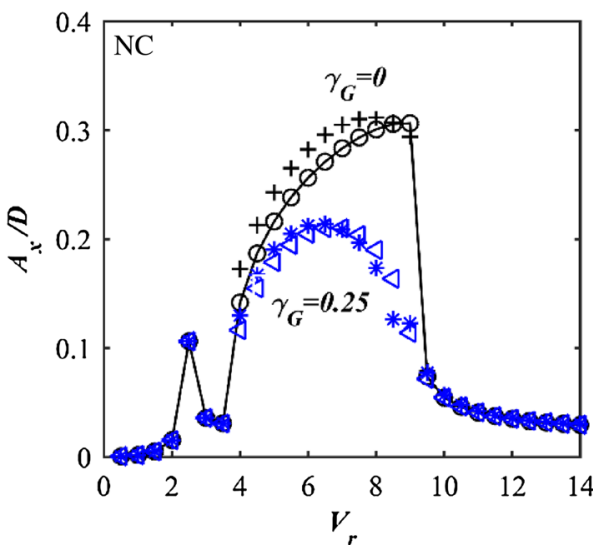

Fig. 3 Comparisons of non-controlled and controlled amplitudes for cylinder with $m^{*}=2.6$ and $\xi=0.00361$ : lines with circles (crosses) and triangles (stars) denote numerical (analytical) results

In the NC case, based on Eqs. (16), (19) and (28), the nonlinearly controlled force and displacement amplitudes with $\omega=\delta=1$ can be simultaneously solved through

$$
q_{M}=\frac{1}{\alpha_{y q}}\left[\left(2 \xi+\frac{\gamma}{\mu}\right) y_{M}+\gamma_{G} \frac{3 y_{M}^{3}}{4}\right],
$$


(a)
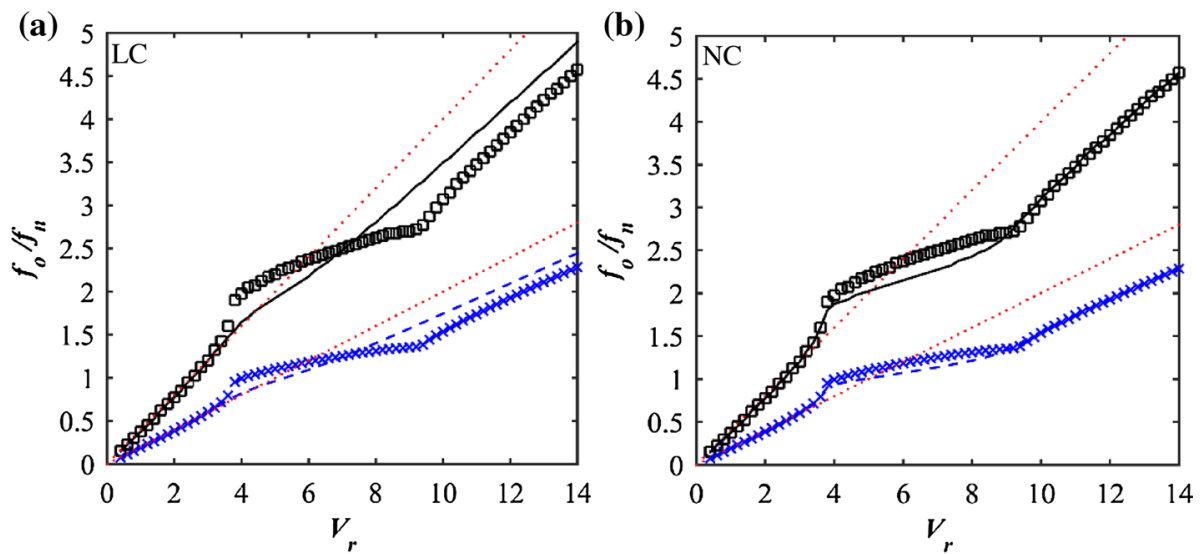

(c)

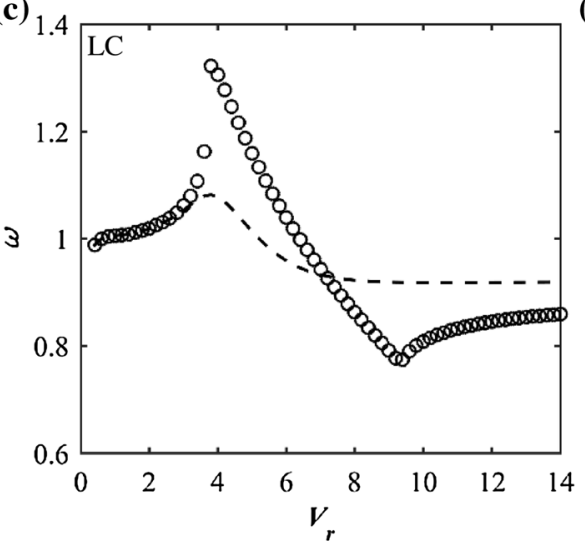

(d)

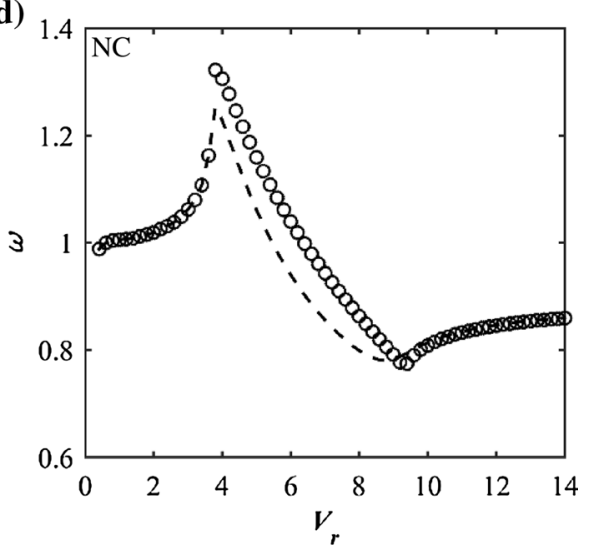

Fig. 4 Comparison of oscillation frequency ratios $(\mathbf{a}, \mathbf{b})$ and resonant frequencies $(\mathbf{c}, \mathbf{d})$ for cylinder with $m^{*}=2.6, \xi=0.00361$ and $\beta_{K}=\gamma_{G}=0.5$ : lines (symbols) denote controlled (non-controlled) results. Higher $x$ and lower $y$ frequencies are shown in (a, b) with Strouhal rule (dotted lines)

$$
\begin{aligned}
y_{M} & =\frac{1}{\beta}\left(\frac{3}{4} \varepsilon \lambda q_{M}^{3}-\varepsilon q_{M}\right), \\
\left(\alpha_{x q} q_{M}^{2}\right)^{2} & =\left[2\left(2 \xi+\frac{\gamma}{\mu}\right) x_{M}\right]^{2}+\left[-4 x_{M}+\left(x_{M}+\alpha_{x} \frac{3 x_{M}^{3}}{4}+\beta_{x} \frac{x_{M} y_{M}^{2}}{2}\right)\right]^{2} .
\end{aligned}
$$

Above LC (Eqs. 29-31) and NC (Eqs. 33-35) systems can be helpful for parametrically investigating the effect of system parameters without performing numerical integrations, as demonstrated in Sect. 4.3. It is also noticed that, for this particular frequency ratio case $(\omega=\delta=1)$, the geometrically nonlinear $\alpha_{y}$ and $\beta_{y}$ terms do not affect the controlled responses since $\left(1-\omega^{2}\right)=0$, whereas the associated $\alpha_{x}$ and $\beta_{x}$ terms do. In the following, the parametric studies are presented and discussed.

\section{Parametric investigation and discussion}

Several aspects in linear and nonlinear active controls of the 2-DOF VIV of circular cylinders with geometric and hydrodynamic nonlinearities are discussed through the cases of varying reduced velocities $\left(0<V_{r}<14\right)$. For the cylinder with a given $m^{*}$ and $\xi$, the control gains $\beta_{K}$ and $\gamma_{G}$ are realistically specified such that their terms have the same order of magnitude as other damping $(\xi, \gamma)$ effects, see Eqs. (25) and (28). Accordingly, it is deduced that $\beta_{K}$ and $\gamma_{G}$ should be of the order of unity. 
(a)

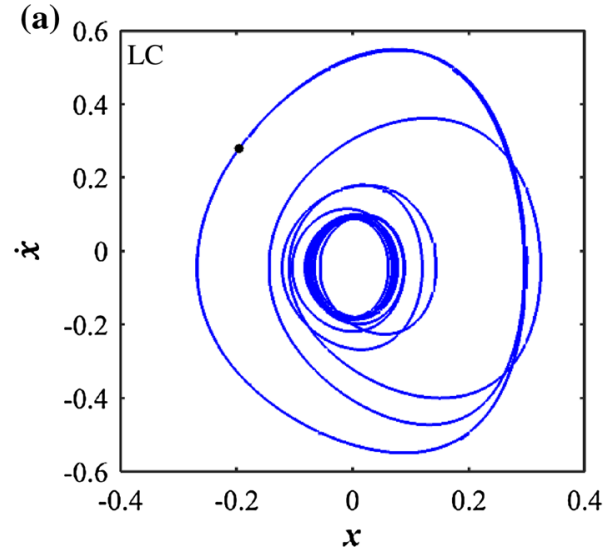

(c)

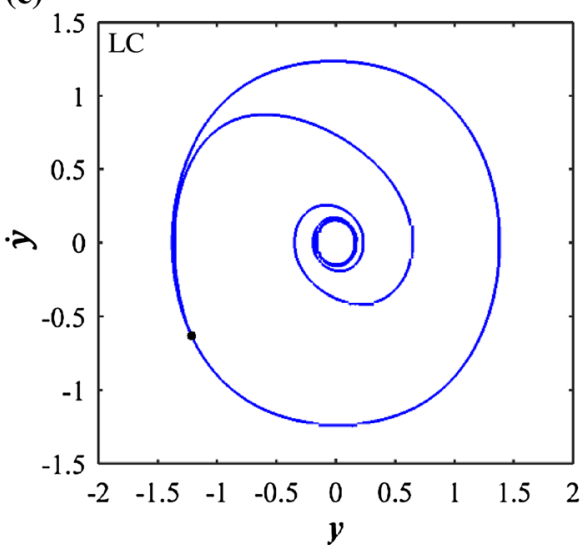

(e)

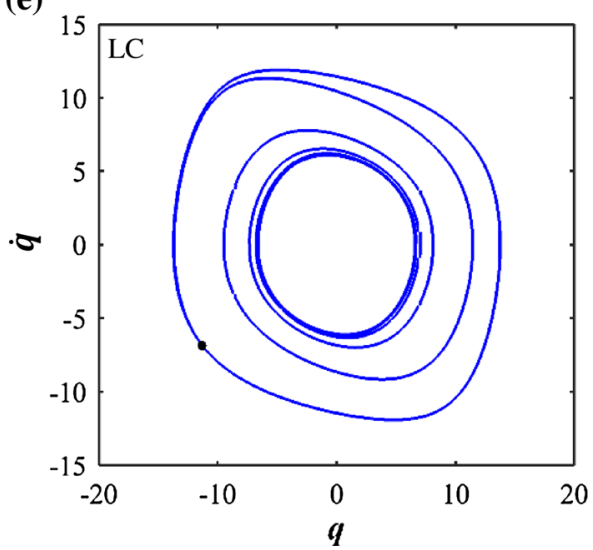

(b)

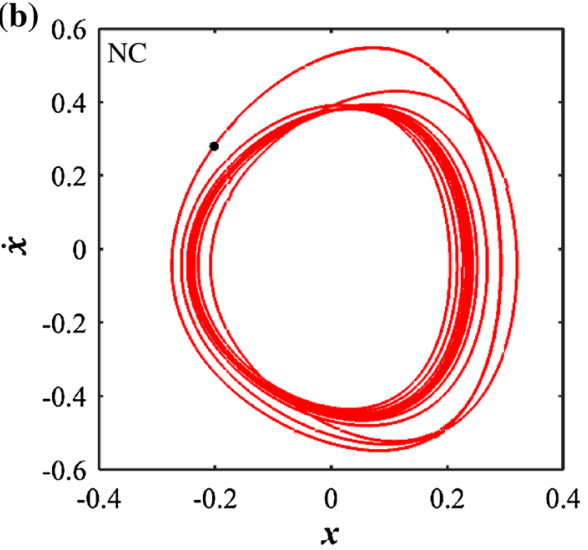

(d)

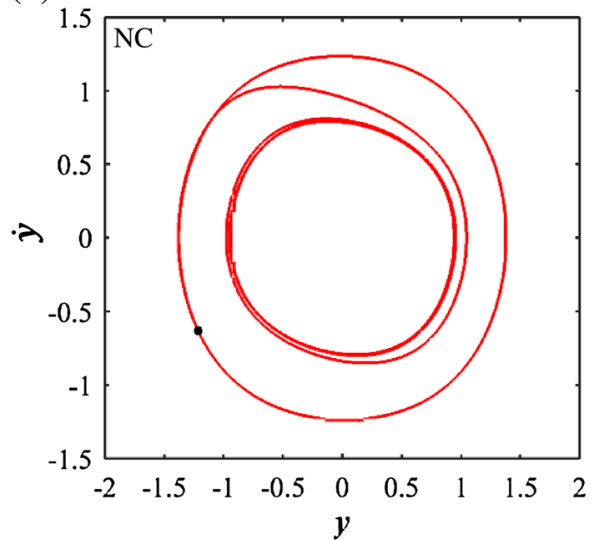

(f)

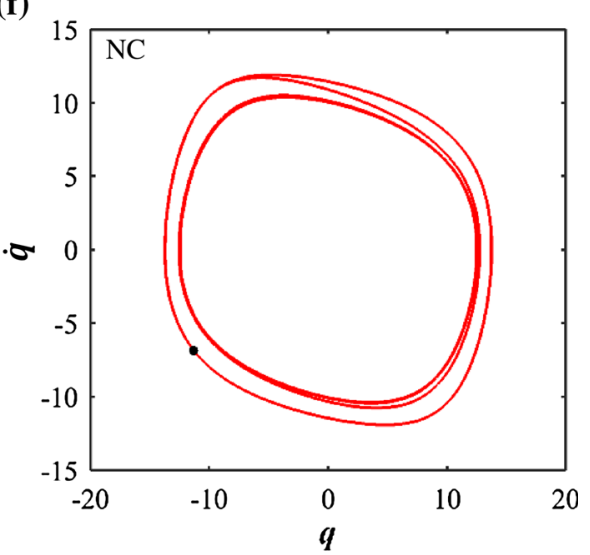

Fig. 5 Phase plane portraits for cylinder with $m^{*}=2.6, \xi=0.00361, \beta_{K}=\gamma_{G}=0.5$ and $V_{r}=7$ : a dot represents the moment of control activation

\subsection{Model calibration and validation}

Because the three nonlinearly coupled equations (3-5) are used for the first time as a 2-DOF VIV prediction model, being the extended version of the linear cylinder model proposed by Bai and Qin [1] and being different from other models with typical four equations [37], a model calibration is required to determine appropriate empirical coefficients $(\varepsilon, \beta)$. This can be achieved by validating the obtained analytical and numerical results with experimental data. In this study, $\varepsilon$ and $\beta$ are tuned such that the dynamic model captures the main lock-in range, maximum cross-flow $\left(A_{y} / D\right)$, and in-line $\left(A_{x} / D\right)$ amplitudes associated with the upper branch, and possible response jump due to the nonlinear hysteresis effect. 
(a)

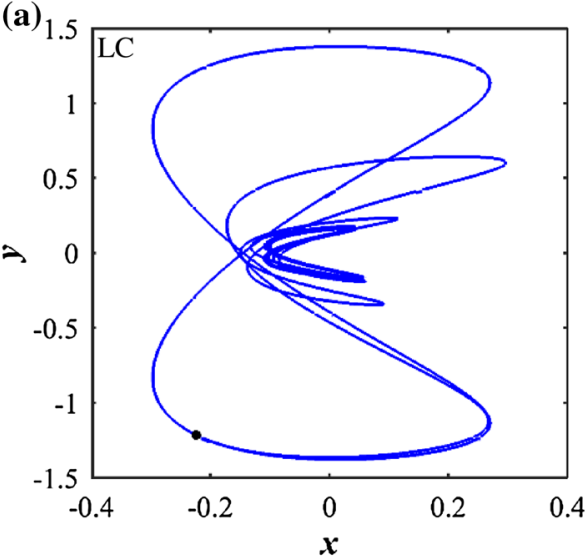

(c)

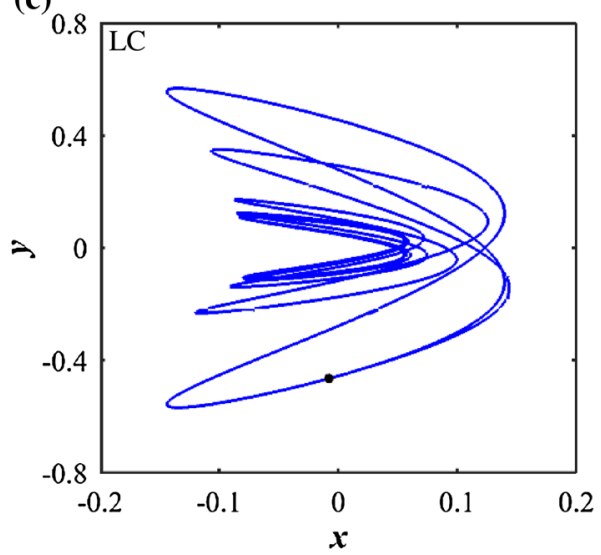

(b)

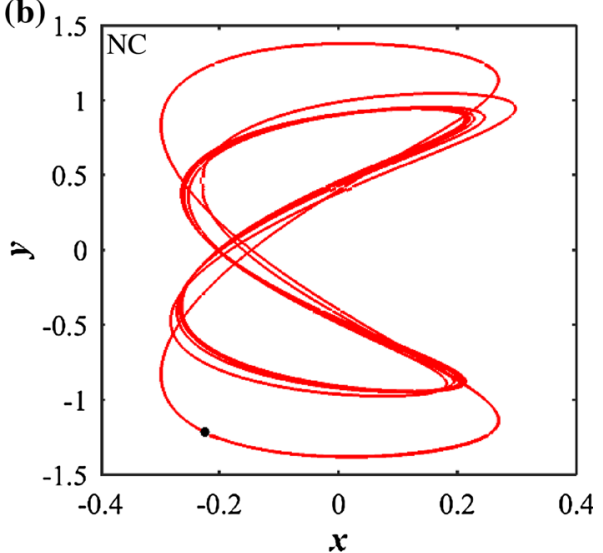

(d)

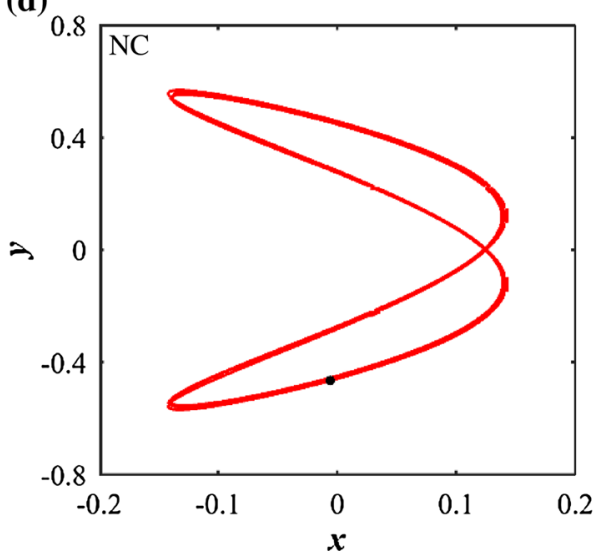

Fig. 6 Two-dimensional $X-Y$ motion trajectories at $V_{r}=7(\mathbf{a}, \mathbf{b})$ and $V_{r}=4(\mathbf{c}, \mathbf{d})$ for cylinder with $m^{*}=2.6, \xi=0.00361$ and $\beta_{K}=\gamma_{G}=0.5$ : a dot represents the moment of control activation

Accordingly, the low-mass-damping 2-DOF cylinder $\left(m^{*}=2.6, \xi=0.00361\right)$ tested by Jauvtis and Williamson [23] is considered. Comparisons of analytical (circles), numerical (triangles) and experimental (squares) results are displayed in Fig. 2 where $A_{x} / D$ (Fig. 2a) and $A_{y} / D$ (Fig. 2b) responses are plotted versus $V_{r}$. The tuning trials are carried out with a criterion by which the predicted maximum cross-flow and in-line amplitudes in the upper branches, as well as the associated lock-in ranges, are matched satisfactorily with the associated experimental results. This is deemed suitable as attention is placed on the most critical response and excitation range for a given $m^{*}$ and $\xi$ [37]. The most satisfactory calibration is found in Fig. 2 with a single set of $\varepsilon=0.058$ and $\beta=12$ : the model predicts the first pure in-line VIV $\left(1.5<V_{r}<3\right)$, the self-limiting maximum amplitudes $\left(A_{y} / D \approx 1.5\right.$ and $\left.A_{x} / D \approx 0.3\right)$ in the main lock-in range $\left(4<V_{r}<10\right)$, and the response jump at $V_{r} \approx 9$. Greater differences in maximum $A_{y} / D$ between analytical and numerical results are also noticed in Fig. $2 \mathrm{~b}$ due to the omitted higher-order harmonic contributions in the analytical solution (Eqs. (6-8)). Nevertheless, such discrepancies are reduced for smaller $A_{x} / D$ as shown in Fig. 2a. The effect of high-order harmonics would become negligible for the controlled system with decreased responses. To also capture the maximum $A_{y} / D$ in the lower branch $\left(V_{r}>8\right)$, another set of $\varepsilon$ and $\beta$ may be introduced. If tuning with different and large set of experimental data is required, an optimization algorithm could also be employed for optimal calibration [7]. Herein, we use $\varepsilon=0.058$ and $\beta=12$ across the $V_{r}$ range.

Since the proposed model can predict the 2-DOF VIV in the absence of control, both LC and NC are now considered. By triggering the active $Y$ control with $\beta_{K}=\gamma_{G}=0.25$ versus the uncontrolled case $\left(\beta_{K}=\gamma_{G}=0\right)$, analytical and numerical $X-Y$ responses are compared in Fig. 3a, c (LC) and in Fig. 3b, d (NC). It can be seen that both $A_{x} / D$ and $A_{y} / D$ are reduced with increasing $\beta_{K}$ and $\gamma_{G}$. The response jumps also disappear. Such suppression improves overall analytical-numerical comparisons enabling almost identical results due to the diminishing effects of high-order harmonics and nonlinearities. With the same control gain $\left(\beta_{K}=\gamma_{G}\right)$, the LC system (Fig. 3a, b) entails a greater reduction in both $X$ and $Y$ responses. This trend is 
(a)

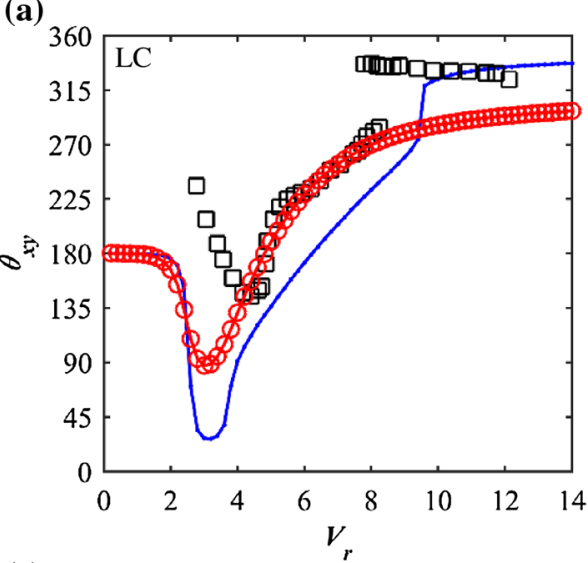

(c)

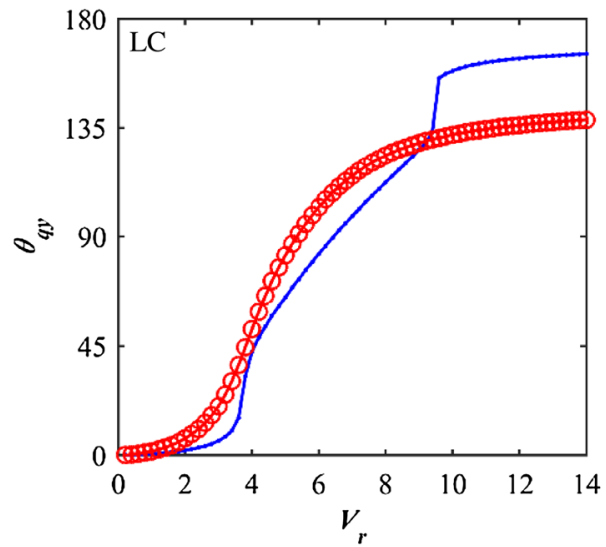

(e)

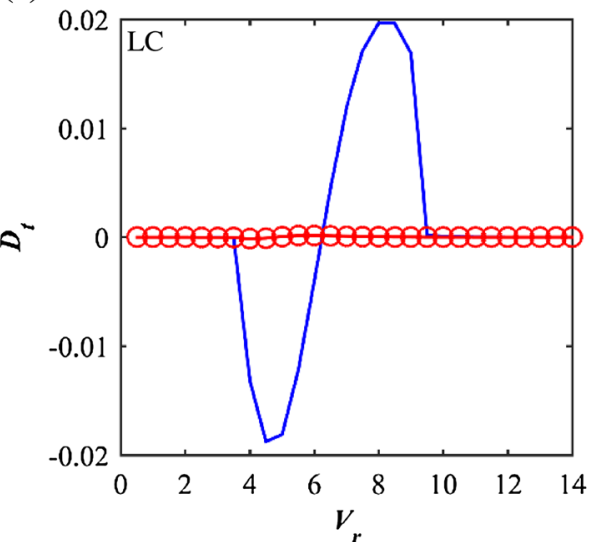

(b)

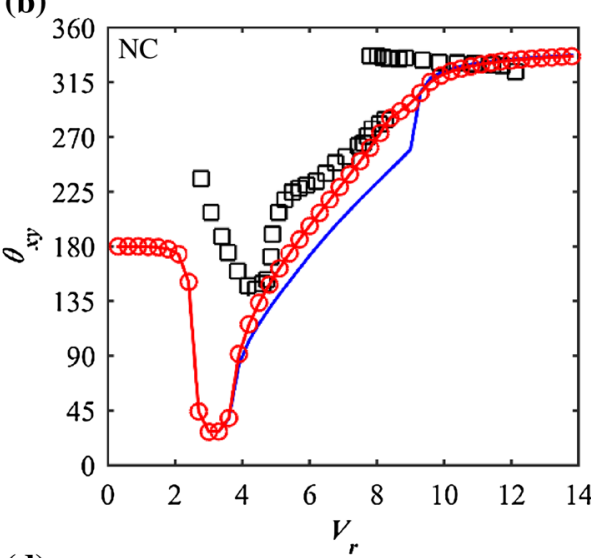

(d)

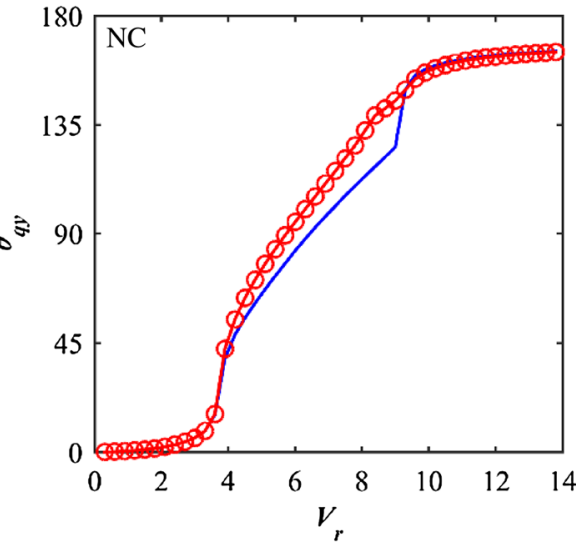

(f)

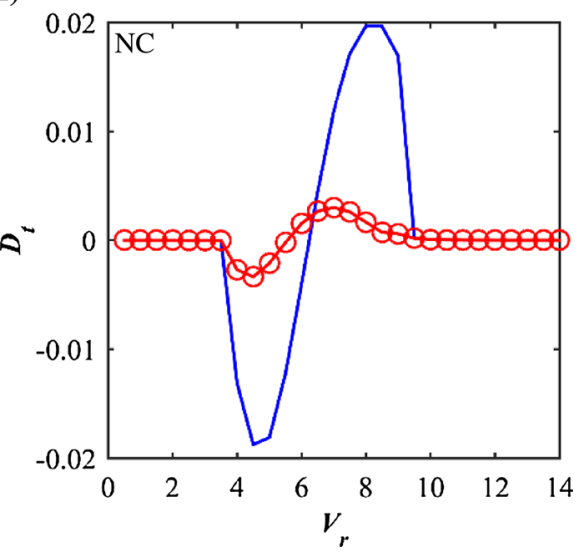

Fig. 7 Comparisons of phase relationships (a-d) and mean drift effect (e, f) for cylinder with $m^{*}=2.6, \xi=0.00361$ and $\beta_{K}=\gamma_{G}=0.5$ : lines (circles) denote non-controlled (controlled) results; squares denote experimental free-vibration data

similar to the CFD study of Mehmood et al. [27] who considered, however, a 1-DOF VIV active control of a much higher $m^{*}=149.10$ and very low $\mathrm{Re}=106$. They showed a greater $A_{y} / D$ suppression by LC. Our focus is placed on the low-mass $\left(m^{*}<6\right)$ cylinder undergoing 2-DOF VIV whose problems are found in a wide range of offshore applications. It should also be noted that the first in-line VIV peak is unaffected by either LC (Fig. 3c) or NC (Fig. 3d) due to the negligible $A_{y} / D$ within that region. Instead, an in-line control could be applied for $1.5<V_{r}<3$. Based on results in Fig. 2 and 3, $\varepsilon=0.058$ and $\beta=12$ are used in the following studies. 
(a)

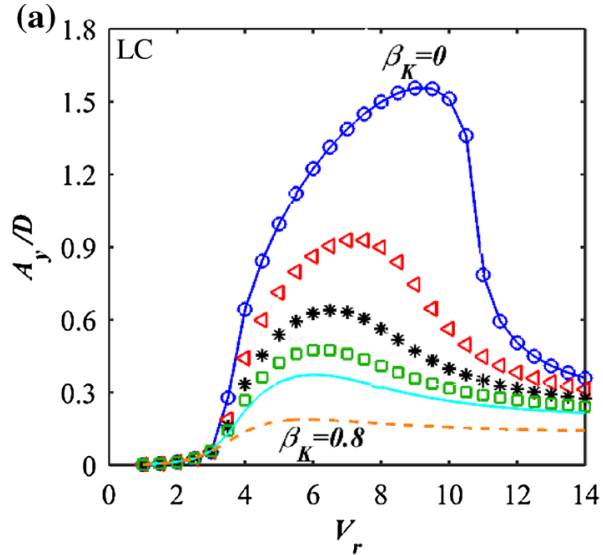

(c)

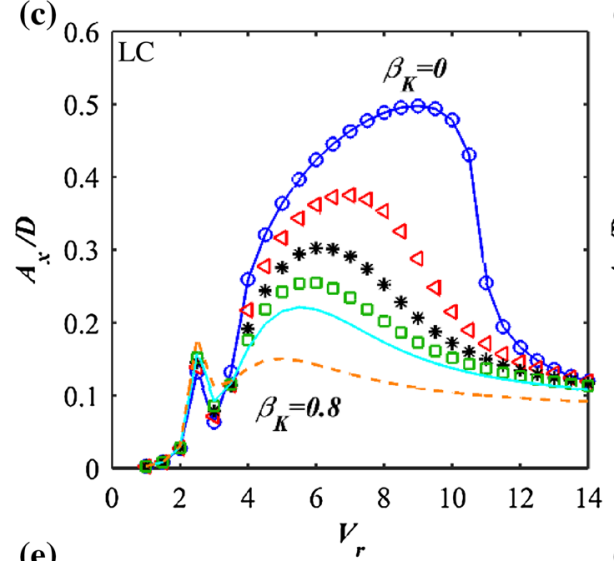

(e)

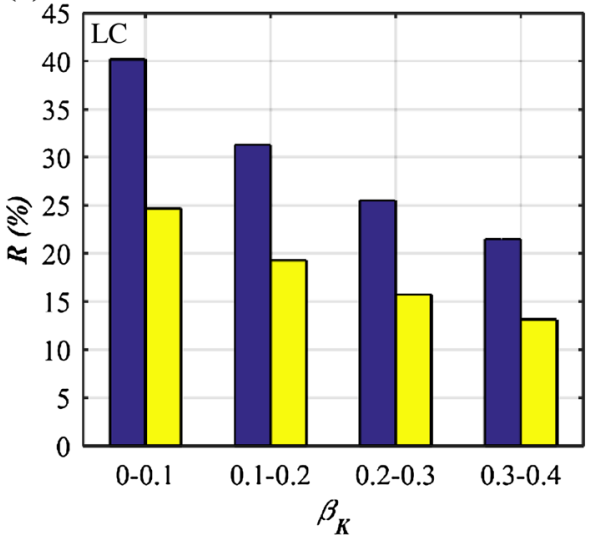

(b) 1.8

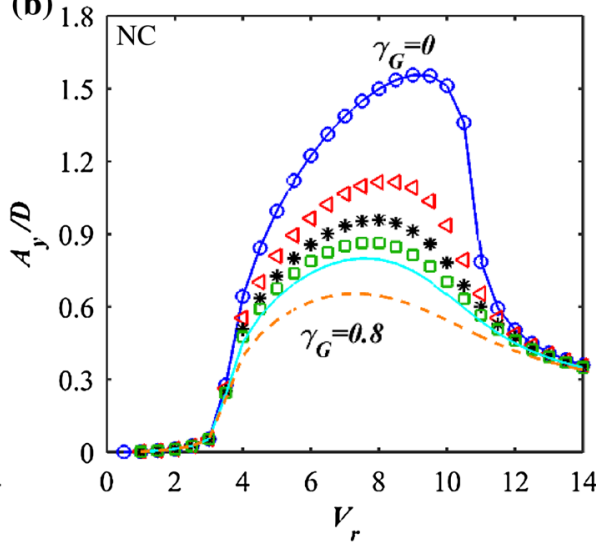

(d)
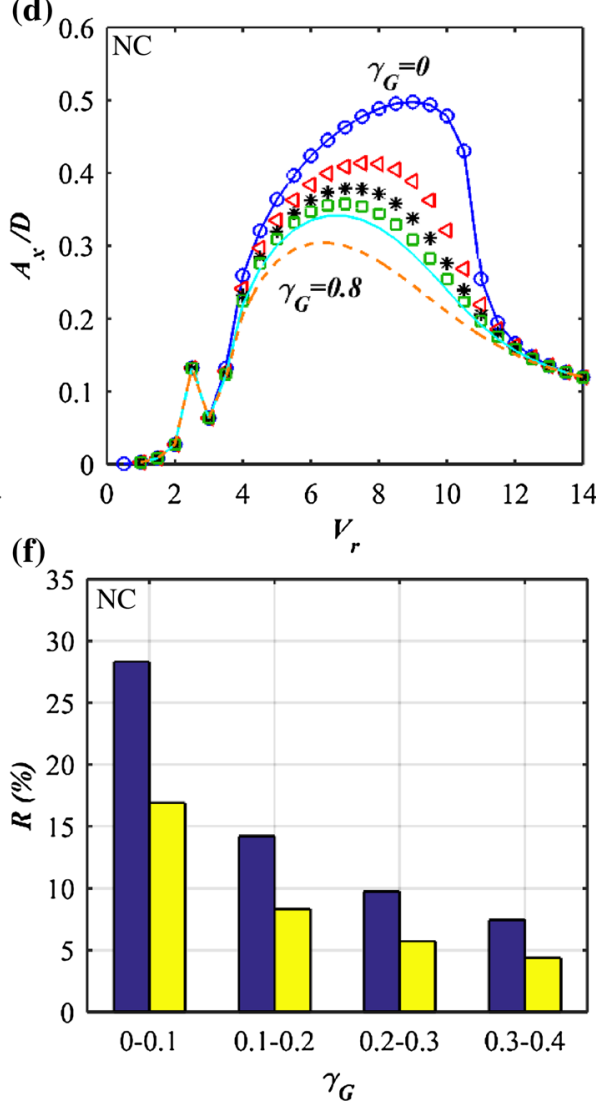

Fig. 8 Influence of control gain on response amplitudes (a-d) and maximum amplitude reduction percentage $R(\mathbf{e}, \mathbf{f})$ for cylinder with $m^{*}=1.2, \xi=0.00361, \beta_{K}$ and $\gamma_{G}$ being increased from 0 to $0.1,0.2,0.3,0.4$ and 0.8 , respectively

\subsection{Controlled frequencies, motion trajectories, phases, and static drifts}

With $m^{*}=2.6$ and $\xi=0.00361$, Fig. 4 presents the cylinder $X-Y$ oscillation frequencies normalized with respect to the natural frequency $\left(f_{0} / f_{\mathrm{n}}\right)$ as well as the resonant frequency normalized with the vortex-shedding frequency $(\omega)$, see Eqs. (6-8), based on LC (Fig. 4a, c) and NC (Fig. 4b, d) with $\beta_{K}=\gamma_{G}=0.5$. Controlled versus non-controlled frequencies are plotted with lines and symbols, respectively, with higher $f_{0} / f_{\mathrm{n}}$ values in Fig. 4a, b corresponding to $X$ responses. It can be seen that, in general, LC has a greater effect on $f_{0} / f_{n}$ and $\omega$ than NC, especially with respect to the upper $\left(4<V_{r}<9\right)$ and lower $\left(V_{r}>9\right)$ branches. With linear control, the $f_{o} / f_{n}$ (Fig. 4a) and $\omega$ (Fig. 4c) trends remarkably deviate from those without a control. The $f_{o} / f_{n}$ plots of the controlled $X-Y$ responses exhibit qualitatively similar features to the Strouhal-based dotted lines 
(a)

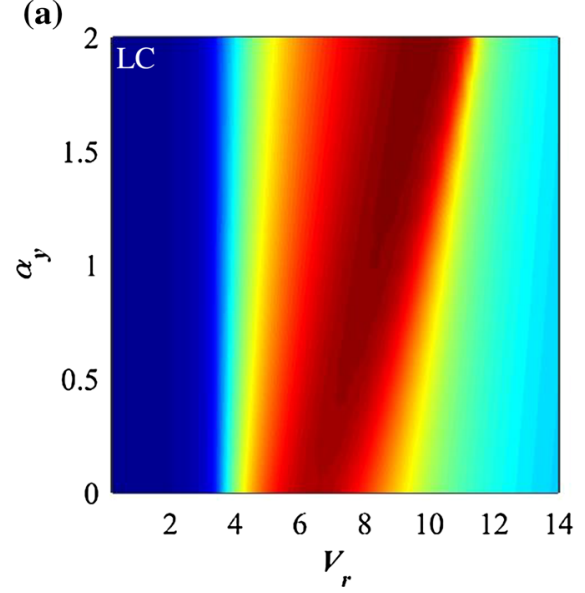

(c)

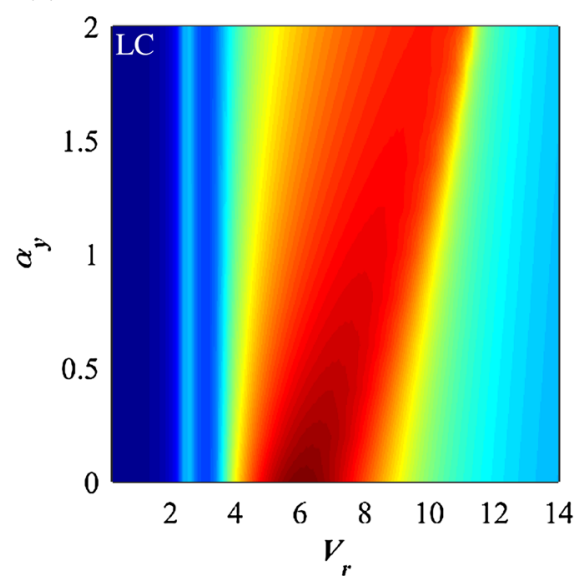

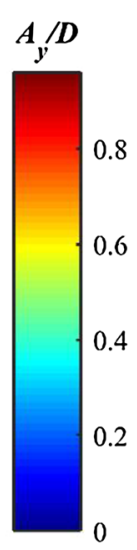

(b)

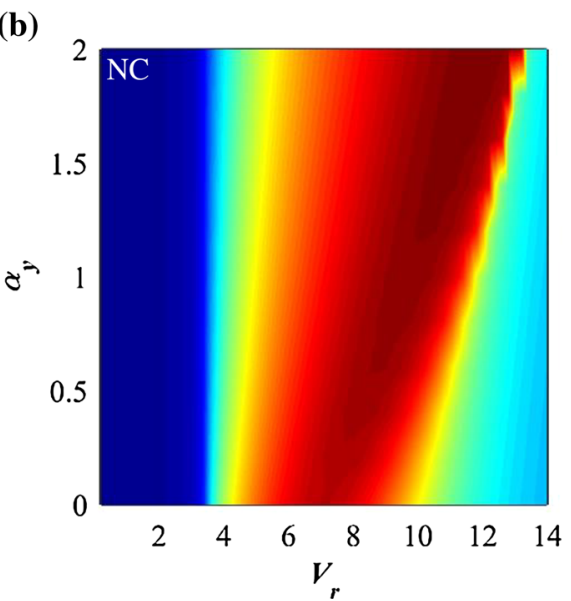

(d)
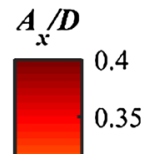

2

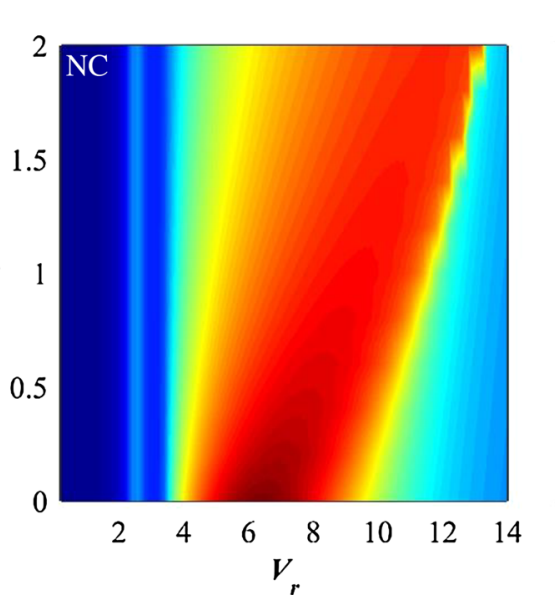

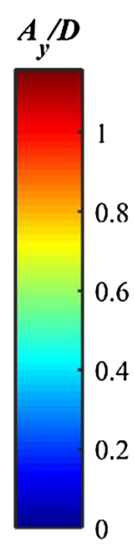

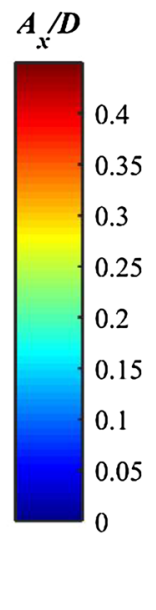

Fig. 9 Influence of geometrically nonlinear coefficient $\left(\alpha_{y}\right)$ on controlled amplitudes for cylinder with $m^{*}=1.2, \xi=0.00361$ and $\beta_{K}=\gamma_{G}=0.1$

whose slopes represent the estimation of the vortex-shedding frequency in cross-flow ( $\mathrm{St} \approx 0.2$ ) and in-line $(\mathrm{St} \approx 0.4)$ directions for a stationary smooth cylinder in subcritical flows [6]. Accordingly, $\omega$ values of the controlled responses become close to the unity, suggesting the desynchronization state [13]. As a result, the associated responses are more reduced; see Fig. 3 a versus $\mathrm{b}\left(A_{y} / D\right)$ and Fig. $3 \mathrm{c}$ versus d $\left(A_{x} / D\right)$. With a higher gain, both LC and $\mathrm{NC}$ would entail a greater departure of $f_{0} / f_{\mathrm{n}}$ and $\omega$, and subsequently greater $X-Y$ amplitude reductions. Nevertheless, the controlled $X-Y$ frequencies in Fig. 4a, b still maintain their dual-resonant 2:1 frequency ratios across the $V_{r}$ range and regardless of the control scheme. This suggests how the associated hydrodynamic added masses are controlled too.

With $\beta_{K}=\gamma_{G}=0.5$ and $V_{r}=7$, the phase plane plots of $x, y$ and $q$ variables are exemplified in Fig. 5 for LC (Fig. 5a, c and e) and NC (Fig. 5b, d and f). By turning on the control actuator (the dots in Fig. 5) after initial transient oscillations, limit cycles of periodic motions are stabilized for overall controlled responses. These plots justify the assumption made in Sect. 3 for which a primary harmonic motion with $\omega(y, q)$ and $2 \omega(x)$ is postulated in Eqs. (6-8). To further visualize dual resonances in the presence of control, Fig. 6 compares $X-Y$ motion trajectories in the case of $V_{r}=7$ (Fig. 6a, b) and $V_{r}=4$ (Fig. 6c, d). It can be seen that, when LC (Fig. 6a, c) or NC (Fig. 6b, d) is activated, the figure-of-eight appearances are still maintained with appreciable repeatability in the last ten cycles shown, and with the two lobs pointing downstream (positive $x$ ) or upstream. Results in Fig. 5 and 6 justify the negligible effect of higher harmonics in the controlled responses.

Apart from the controlled $X-Y$ amplitudes, frequencies, and trajectories, it is also of interest to evaluate the associated phase differences $\theta_{x y}$ and $\theta_{q y}$, the latter implying the fluid-cylinder energy transfer [41]. With $\beta_{K}=\gamma_{G}=0.5$, numerical results (circles) of $\theta_{x y}$ and $\theta_{q y}$ are plotted in Fig. 7a, c for LC and in Fig. 7b, d 

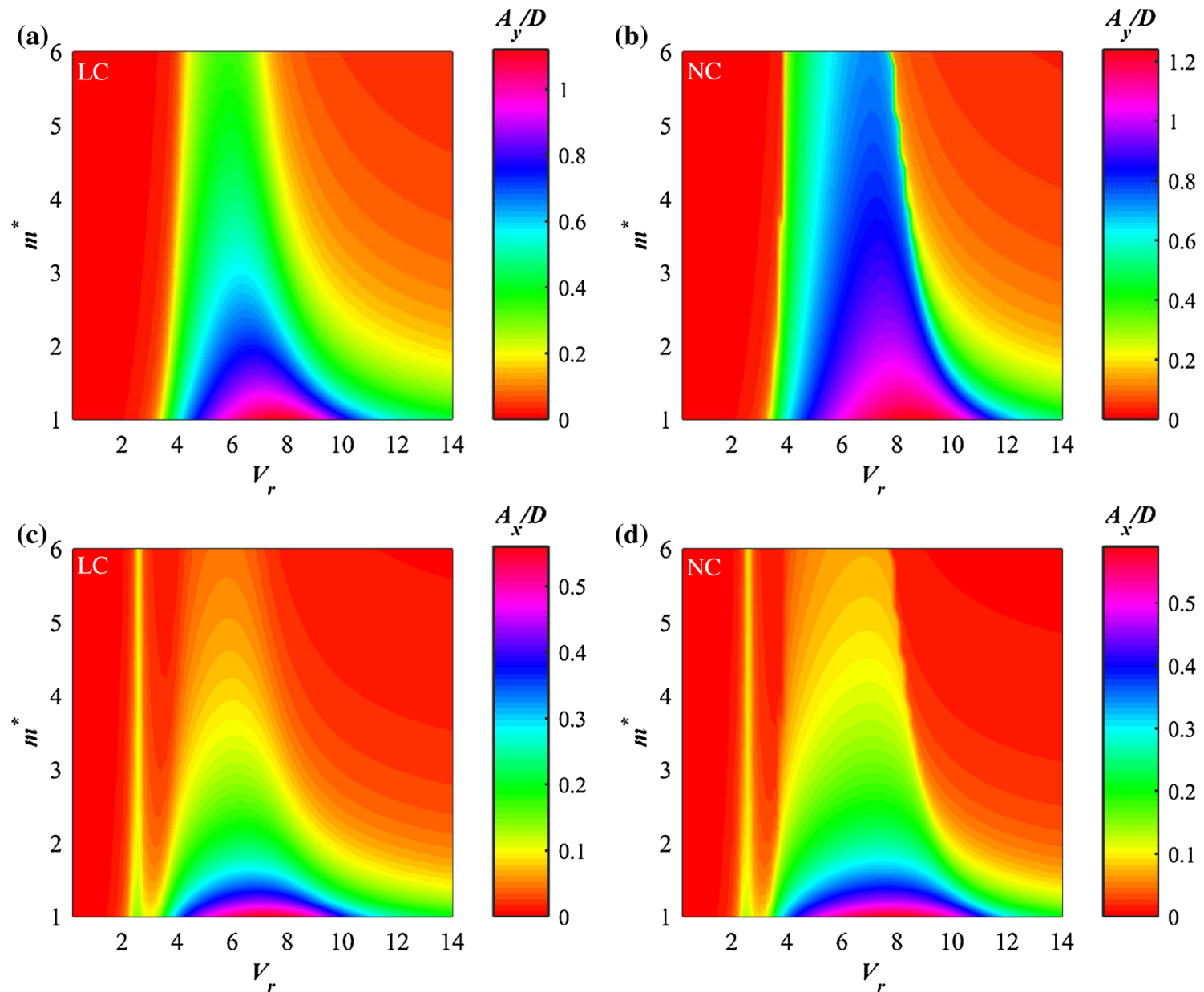

Fig. 10 Influence of $m^{*}$ on controlled amplitudes for cylinder with $\xi=0.00361$ and $\beta_{K}=\gamma_{G}=0.1$

for NC, respectively. Experimental $\theta_{x y}$ data (squares) of Jauvtis and Williamson [23] and numerical $\left(\theta_{x y}, \theta_{q y}\right)$ results (solid lines) in the absence of control are also overlapped in Fig. 7a-d. With varying $V_{r}$, numerical and experimental results reveal similarly the referenced $\pi / 2<\theta_{x y}<2 \pi$ and $\pi / 4<\theta_{q y}<\pi$ within the main excitation range of $4<V_{r}<14$ covering initial, upper and lower branches, and the observed jump in the phase responses at $8<V_{r}<10$. The $\theta_{x y}$ range suggests a transition from the clockwise figureof-eight trajectories $\left(\pi / 2<\theta_{x y}<3 \pi / 2\right)$ to the anticlockwise ones $\left(3 \pi / 2<\theta_{x y}<2 \pi\right)$ with increasing $V_{r}$, whereas the $\theta_{q y}$ range suggests the fluid excitation (as opposed to the damping) leading to VIV responses. With LC, a jump disappears from Fig. 7c, and the $\theta_{q y}$ phase change is clearly observed for $V_{r}>9$ which gives rise to $\pi / 4<\theta_{q y}<3 \pi / 4$. With NC, the jump also disappears from Fig. $7 \mathrm{~d}$ although $\theta_{q y}$ values are less affected. In both control cases, the figure-of-eight patterns maintain their clockwise or anticlockwise shapes since $\theta_{x y}$ values in both Fig. $7 \mathrm{a}, \mathrm{b}$ slightly change. This is in agreement with the results in Fig. 6.

The capability of suppressing the static drift due to the geometric nonlinear coupling can be illustrated in Fig. 7e (LC) and $\mathrm{f}(\mathrm{NC})$ with $\beta_{K}=\gamma_{G}=0.5$. The drift, which changes its sign following $\theta_{x y}$ in Eq. (20) (Fig. 7a, b), is almost totally eliminated with LC. This observation is hopeful since passive VIV control devices such as strakes generally have a limitation in the mitigation of in-line force including its amplified mean component. The active cylinder control might provide an alternative strategy to suppress the mean drag effect, and this can be the subject of future study using a more complete three-dimensional flexible cylinder model [42]. 

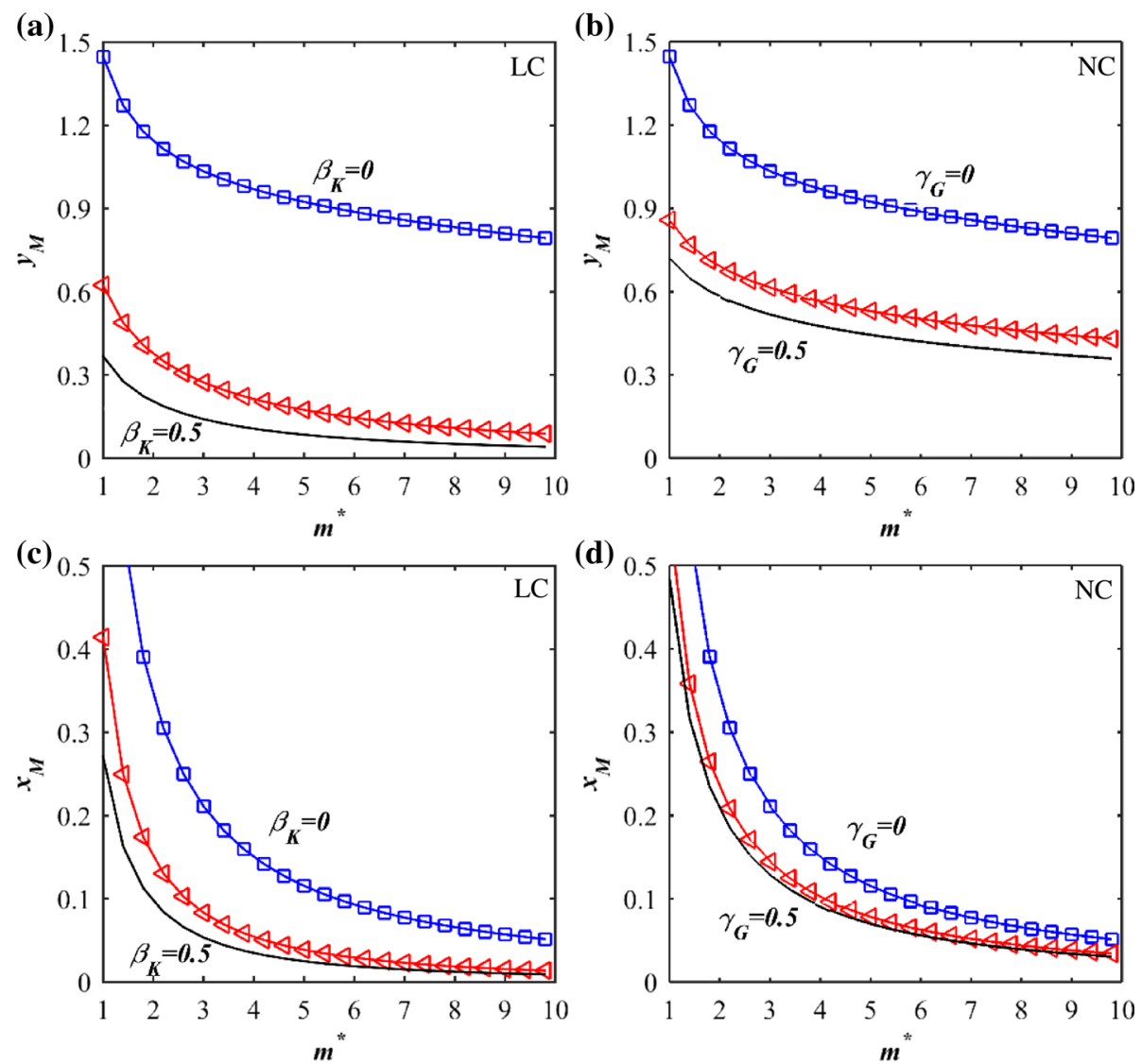

Fig. 11 Comparisons of response amplitudes at ideal perfect lock-in condition with varying $m^{*}$ for cylinder with $\xi=0.00361$, $\beta_{K}$ and $\gamma_{G}$ being increased from 0 to $0.25,0.5$, respectively

\subsection{Influence of control gain, geometrically nonlinear coupling, and mass ratio}

With $m^{*}=1.2$ and $\xi=0.00361$, the controller performance in the reduction of 2-DOF amplitudes is now displayed in Fig. 8 by varying $\beta_{K}$ or $\gamma_{G}$ from 0 to $0.1,0.2,0.3,0.4$ and 0.8 . For LC, amplitudes are suppressed with increasing $\beta_{K}$, leading to a large reduction up to about 88 and $70 \%$ for maximum $A_{y} / D$ (Fig. 8a) and $A_{x} / D$ (Fig. 8c), respectively. Likewise, the NC demonstrates the controlling effect on the 2-DOF VIV with about 58\% $\left(A_{y} / D\right)$ and 39\% $\left(A_{x} / D\right)$ maximum amplitude reductions (Fig. 8b, d). However, the lock-in region does not shift since the mass ratio $m^{*}$ is fixed. Similar qualitative behaviors can be found through a 1-DOF wake oscillator model of Dai et al. [12] where the control gain of a time-delay feedback controller was increased, yielding the decreased $A_{y} / D$. As previously discussed, the response suppression is attributed to the added damping effect with increasing gain. To achieve greater control performance, both $A_{y} / D$ and $A_{x} / D$ can be further reduced with increasing $\beta_{K}$ and $\gamma_{G}$.

The variation of control gain was also employed by Baz and Ro [4] where a 1-DOF direct velocity feedback control was shown to suppress VIV. According to their experiments, the controller would achieve a maximum gain and amplitude reduction. This is also demonstrated in Fig. 8a-d where the increases in $\beta_{K}$ and $\gamma_{G}$ lead to the minimum reduced responses. The histogram plots in Fig. 8e (LC) and $\mathrm{f}(\mathrm{NC})$ illustrate the maximum amplitude reduction percentage $(R)$ of both cross-flow (left blue bar) and in-line (right yellow bar) responses when increasing consecutively the gain with each 0.1 increment (i.e., from 0 to 0.1 , from 0.1 to 0.2 and so on). It can be observed that both $X-Y$ responses and both LC/NC demonstrate a gradual decreasing $R$ as each gain increment is applied. This confirms the existence of maximum gain value and, thus, the maximum amplitude reduction capability for each controller. The feedback closed-loop control will reach a maximum efficiency once the control gain is sufficiently large [22].

The effect of geometric displacement coupling is now highlighted in Fig. 9 where $\alpha_{y}$ governing $y^{3}$ in Eq. (4) is varied for a given cylinder $\left(m^{*}=1.2, \xi=0.00361\right)$ and control gain $\left(\beta_{K}=\gamma_{G}=0.1\right)$. Contour plots of 
(a)

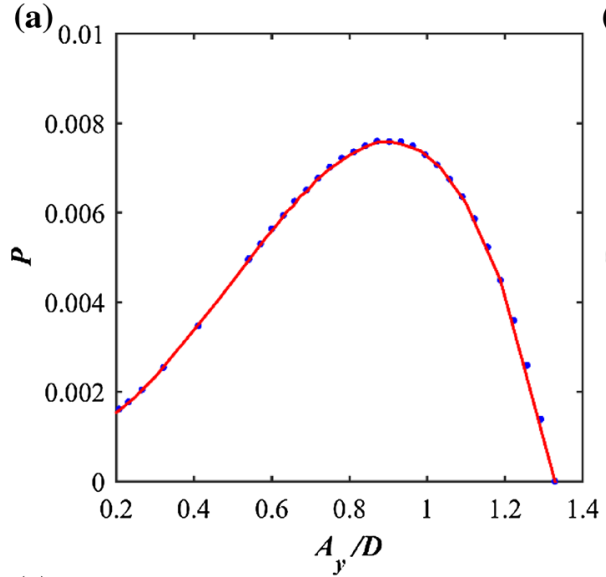

(c)

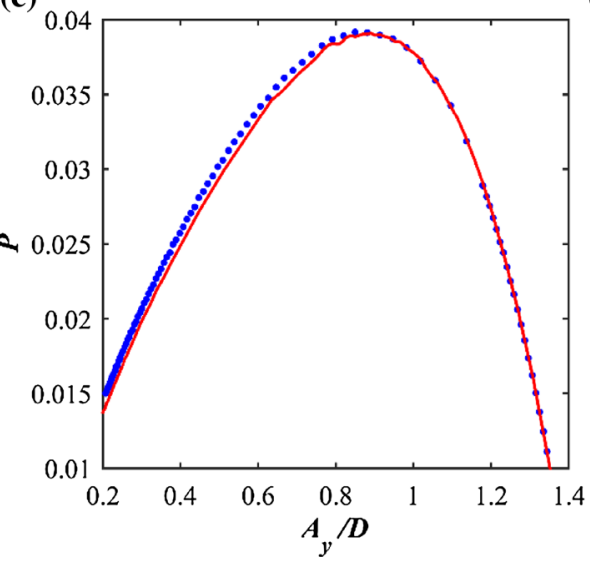

(b)

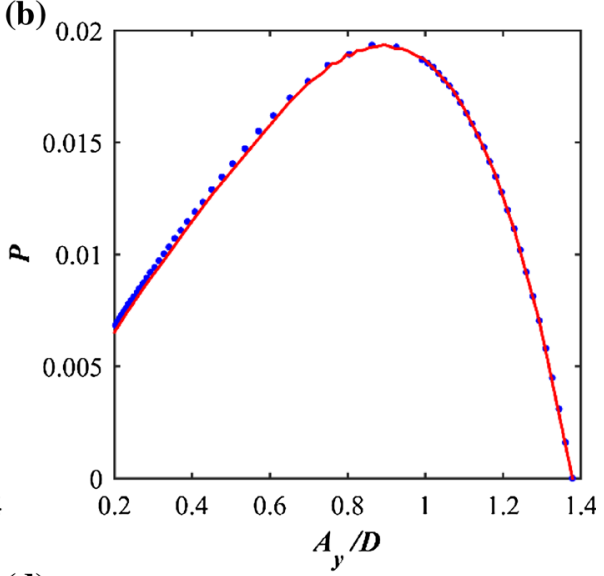

(d)

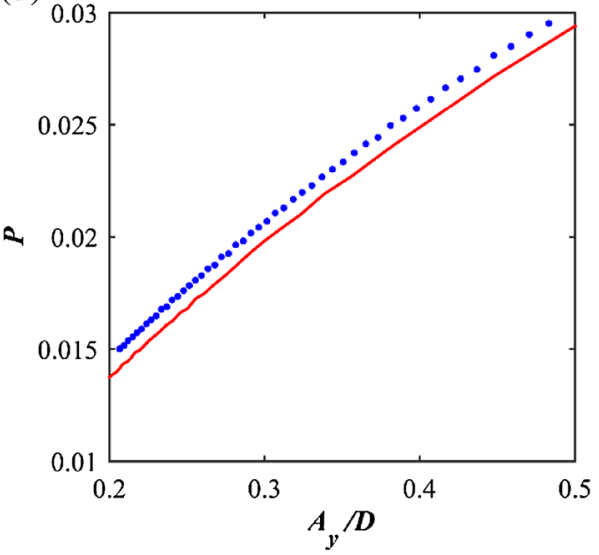

Fig. 12 Comparisons of averaged power requirement with the targeted reduced amplitudes with LC (dots) versus NC (lines): a $m^{*}=2.6$ and $\xi=0.00361, \mathbf{b} m^{*}=6.9$ and $\xi=0.00361, \mathbf{c} m^{*}=1$ and $\xi=0 ; \mathbf{d}$ showing the zoomed results in $\mathbf{c}$

the suppressed 2-DOF amplitudes with varying $\alpha_{y}$ and $V_{r}$ are displayed in Fig. 9a and c for LC and in Fig. 9b and $\mathrm{d}$ for NC. It can be seen how the increased cylinder nonlinearities affect the main lock-in responses which exhibit the right-bending features and widening resonance ranges due to the enhanced cubic nonlinearities in both $A_{y} / D$ (Fig. 9a, b) and $A_{x} / D$ (Fig. 9c, d) plots. Owing to the intrinsic $X-Y$ coupling, maximum $A_{y} / D$ are slightly increased, while maximum $A_{x} / D$ are more decreased as $\alpha_{y}$ is increased for both LC/NC. This energy transfer between the cylinder $X-Y$ motions, leading to the opposite increasing vs. decreasing responses, might be useful in some applications such as cylinders placed in a tandem where greater in-line VIV reduction of the front cylinder is required. As for other geometric nonlinear terms $\left(\beta_{y}, \alpha_{x}, \beta_{x}\right)$, they have a lesser effect on the controlled responses due to the smaller contributions from the $x$-based $\left(y x^{2}, x^{3}, x y^{2}\right)$ terms.

With $\beta_{K}=\gamma_{G}=0.1$, contour plots of the suppressed 2-DOF amplitudes $\left(A_{y} / D, A_{x} / D\right)$ with varying $m^{*}$ and $V_{r}$ are displayed in Fig. 10. Apart from the fact that the first resonance in-line VIV region is not affected by any $Y$-controllers, Fig. 10 shows how the controlled cylinder with lower $m^{*}$ has greater $X-Y$ responses and lock-in ranges regardless of LC or NC. This is expected from the $m^{*}$ effect viewpoint. The effect of $m^{*}$ on the controlled responses $\left(Y_{M}, X_{M}\right)$ is further highlighted in Fig. 11 with the advantage of analytical expressions in Sect. 3 which has been derived for the referenced resonant lock-in condition $(\omega=\delta=1)$. Two gain values with $\beta_{K}=\gamma_{G}=0.25$ (triangles) and 0.50 (solid lines) are considered for both LC (Fig. 11a, c) and NC (Fig. $11 \mathrm{~b}, \mathrm{~d}$ ) versus the no-control (squares) case $\left(\beta_{K}=\gamma_{G}=0\right)$. In general, both linearly and nonlinearly controlled 2-DOF responses exhibit similar trends of decreasing responses with increasing gain and $m^{*}$. The amplitude reduction function appears to be more nonlinear in a lower mass ratio range $\left(m^{*}<6\right)$. This could emphasize, through an active control study, the effect of 2-DOF VIV on the low-mass cylinder. Nevertheless, due to the limiting control performance, the amplitude reduction capability is reduced from $\beta_{K}=\gamma_{G}=0$ to 0.25 and from $\beta_{K}=\gamma_{G}=0.25$ to 0.5 . 


\subsection{Comparison of power requirement}

Finally, it is of practical importance to examine the power requirement for each controller since the control performance is also dependent on the forces exerted from the controllers, besides the capability of amplitude reduction. Based on the numerically obtained steady-state responses, the dimensionless averaged power $P$ for linear $\left(\beta_{K}\right)$ and nonlinear $\left(\gamma_{G}\right) Y$ control may be evaluated, respectively, from [27]

$$
P=\lim _{t \rightarrow \infty} \int_{0}^{\tau} \beta_{K} \dot{y}^{2}(\tau) \mathrm{d} \tau \quad \text { or } \quad P=\lim _{t \rightarrow \infty} \int_{0}^{\tau} \gamma_{G} \dot{y}^{4}(\tau) \mathrm{d} \tau .
$$

This can be evaluated for a particular number of oscillation periods (herein equal to 100 cycles). The power demand would be particularly useful, e.g., for sizing the control actuators to handle the power requirement. Since the force is applied in the $Y$ direction, the power is dependent on the cylinder $Y$, rather than $X$, velocity.

Depending on the level of targeted controlled amplitudes, Fig. 12 illustrates comparisons of $P$ based on LC (dots) versus NC (lines) for the cylinder with $m^{*}=6.9$ and $\xi=0.00361$ (Fig. 12a), $m^{*}=2.6$ and $\xi=0.00361$ (Fig. 12b) and the limiting case of $m^{*}=1$ and $\xi=0$ (Fig. 12c). It can be seen that both LC and NC generally require comparable $P$ for both high (Fig. 12a) and low (Fig. 12b) $m^{*}$ as well as in both high $\left(A_{y} / D>0.9\right.$ ) and low $\left(A_{y} / D<0.9\right)$ amplitude ranges, with the maximum $P$ occurs at the targeted $A_{y} / D \approx 0.9$. Based solely on the maximum amplitude reduction, the LC appears to be superior to NC. However, for the neutrally buoyant cylinder (Fig. 12c), the NC system is seen to require a lower $P$ (about 5\%) within the lower amplitude range as zoomed in Fig. 12d. This 2-DOF control observation is similar to the CFD control results of Mehmood et al. [27] who suggested a better NC performance when the targeted $A_{y} / D<0.2$ for a very high $m^{*}=149.1$ of the 1-DOF circular cylinder.

\section{Conclusions}

Investigation into the effectiveness of active linear and nonlinear controls by using the cylinder transverse velocity feedback for the suppression of two-dimensionally coupled VIV of a flexibly mounted circular cylinder in uniform flows has been presented. The reduced-order nonlinear dynamic model simulating the cylindervortex strength interaction is based on the use of coupled Duffing-Rayleigh oscillators which capture basic VIV phenomena in the absence of control. Model empirical coefficients have been calibrated with free-vibration experimental data and then applied to the parametric studies with active controls. Combined analytical and numerical results are presented and discussed. At lock-in oscillation frequencies, some analytical expressions for response amplitudes have been derived to explicitly describe the resonant dynamics.

By focusing on the main synchronization range and system dimensionless parameters, the actively controlled responses have been assessed in terms of response amplitudes, oscillation frequencies, phase relationships, orbital motions and control power requirements in a wide range of reduced velocities. The effects of control gain, mass ratio and geometric nonlinear displacement have also been explored. Parametric results reveal that linear and nonlinear controllers can be implemented for mitigating the coupled cross-flow/in-line VIV and the associated static drifts. Despite both control systems modify the oscillation frequencies and phase relations, the suppressed two-dimensional responses maintain dual 2:1 resonance features with figure-of-eight orbital motions. Depending on the mass-damping ratio, reduced velocity, control gain, and targeted controlled amplitude, the linear control generally provides a better performance. However, for the limiting case of neutrally buoyant cylinder with negligible structural damping, the nonlinear control requires lower power in the targeted small-amplitude range. Experimental studies are needed to justify such predictions. The Reynolds number dependence of response amplitudes should also be recognized in the prediction model through, e.g., the wake oscillator coefficients regulating the self-limiting and fluid-structure interaction terms. This will be considered in our future investigations. In addition, the present model may be applied to control a three-dimensional VIV of a long flexible cylinder. This can be achieved, e.g., by embedding the smart sensors within the cylinder fabric at locations where potential vibration modes are excited, and then signaling the responses to the actuators for actively controlling the multimode and multi-DOF VIV. 
you give appropriate credit to the original author(s) and the source, provide a link to the Creative Commons license, and indicate if changes were made.

\section{References}

1. Bai, X., Qin, W.: Using vortex strength wake oscillator in modelling of vortex induced vibrations in two degrees of freedom. Eur. J. Mech. B/Fluids 48, 165-173 (2014)

2. Bao, Y., Huang, C., Zhou, D., Tu, J., Han, Z.: Two-degree-of-freedom flow-induced vibrations on isolated and tandem cylinders with varying natural frequency ratios. J. Fluids Struct. 35, 50-75 (2012)

3. Baz, A., Kim, M.: Active modal control of vortex-induced vibrations of a flexible cylinder. J. Sound Vib. 165, 69-84 (1993)

4. Baz, A., Ro, J.: Active control of flow-induced vibrations of a flexible cylinder using direct velocity feedback. J. Sound Vib. 146, 33-45 (1991)

5. Bearman, P.W.: Vortex shedding from oscillating bluff bodies. Annu. Rev. Fluid Mech. 16, 195-222 (1984)

6. Blevins, R.D.: Flow-Induced Vibrations. Van Nostrand Reinhold, New York (1990)

7. Bódai, T., Srinil, N.: Performance analysis and optimization of a box-hull wave energy converter concept. Renew. Energy 81, 551-565 (2015)

8. Chen, W.-L., Xin, D.-B., Xu, F., Li, H., Ou, J.-P., Hu, H.: Suppression of vortex-induced vibration of a circular cylinder using suction-based flow control. J. Fluids Struct. 42, 25-39 (2013)

9. Chen, Z., Fan, B., Zhou, B., Aubry, N.: Control of vortex shedding behind a circular cylinder using electromagnetic forces. Mod. Phys. Lett. B 19, 1627-1630 (2005)

10. Dahl, J., Hover, F., Triantafyllou, M.: Two-degree-of-freedom vortex-induced vibrations using a force assisted apparatus. J. Fluids Struct. 22, 807-818 (2006)

11. Dahl, J.M., Hover, F.S., Triantafyllou, M.S., Oakley, O.H.: Dual resonance in vortex-induced vibrations at subcritical and supercritical Reynolds numbers. J. Fluid Mech. 643, 395-424 (2010)

12. Dai, H.L., Abdelkefi, A., Wang, L., Liu, W.B.: Time-delay feedback controller for amplitude reduction in vortex-induced vibrations. Nonlinear Dyn. 80, 59-70 (2015)

13. Facchinetti, M.L., De Langre, E., Biolley, F.: Coupling of structure and wake oscillators in vortex-induced vibrations. J. Fluids Struct. 19, 123-140 (2004)

14. Filler, J.R., Marston, P., Mih, W.: Response of the shear layers separating from a circular cylinder to small-amplitude rotational oscillations. J. Fluid Mech. 231, 481-499 (1991)

15. Fujisawa, N., Takeda, G.: Flow control around a circular cylinder by internal acoustic excitation. J. Fluids Struct. 17, 903-913 (2003)

16. Gabbai, R.D., Benaroya, H.: An overview of modeling and experiments of vortex-induced vibration of circular cylinders. J. Sound Vib. 282, 575-616 (2005)

17. Govardhan, R.N., Williamson, C.H.K.: Defining the 'modified Griffin plot' in vortex-induced vibration: revealing the effect of Reynolds number using controlled damping. J. Fluid Mech. 561, 147-180 (2006)

18. Hartlen, R.T., Currie, I.G.: Lift-oscillator model of vortex-induced vibration. J. Eng. Mech. 96, 577-591 (1970)

19. Hasheminejad, S.M., Rabiee, A.H., Jarrahi, M., Markazi, A.H.D.: Active vortex-induced vibration control of a circular cylinder at low Reynolds numbers using an adaptive fuzzy sliding mode controller. J. Fluids Struct. 50, 49-65 (2014)

20. Hsiao, F.B., Shyu, J.Y.: Influence of internal acoustic excitation upon flow passing a circular cylinder. J. Fluids Struct. 5, 427-442 (1991)

21. Huang, X.Y.: Feedback control of vortex shedding from a circular cylinder. Exp. Fluids 20, 218-224 (1996)

22. Inman, D.J.: Vibration with Control. Wiley, New York (2006)

23. Jauvtis, N., Williamson, C.: The effect of two degrees of freedom on vortex-induced vibration at low mass and damping. J. Fluid Mech. 509, 23-62 (2004)

24. Korkischko, I., Meneghini, J.R.: Suppression of vortex-induced vibration using moving surface boundary-layer control. J. Fluids Struct. 34, 259-270 (2012)

25. Kovacic, I., Brennan, M.J.: The Duffing Equation: Nonlinear Oscillators and Their Behaviour. Wiley-Blackwell, New York (2011)

26. Low, Y.M., Srinil, N.: VIV fatigue reliability analysis of marine risers with uncertainties in the wake oscillator model. Eng. Struct. 106, 96-108 (2016)

27. Mehmood, A., Abdelkefi, A., Akhtar, I., Nayfeh, A., Nuhait, A., Hajj, M.: Linear and nonlinear active feedback controls for vortex-induced vibrations of circular cylinders. J. Vib. Control 20, 1137-1147 (2014)

28. Nayfeh, A.H.: Introduction to Perturbation Techniques. Wiley, New York (1993)

29. Owen, J.C., Bearman, P.W., Szewczyk, A.A.: Passive control of VIV with drag reduction. J. Fluids Struct. 15, 597-605 (2001)

30. Pontaza, J.P., Chen, H.-C.: Three-dimensional numerical simulations of circular cylinders undergoing two degree-of-freedom vortex-induced vibrations. J. Offshore Mech. Arct. Eng. 129, 158-164 (2006)

31. Quadrante, L.A.R., Nishi, Y.: Amplification/suppression of flow-induced motions of an elastically mounted circular cylinder by attaching tripping wires. J. Fluids Struct. 48, 93-102 (2014)

32. Sarpkaya, T.: Vortex-induced oscillations: a selective review. J. Appl. Mech. 46, 241-258 (1979)

33. Sarpkaya, T.: A critical review of the intrinsic nature of vortex-induced vibrations. J. Fluids Struct. 19, 389-447 (2004)

34. Skop, R.A., Balasubramanian, S.: A new twist on an old model for vortex-excited vibrations. J. Fluids Struct. 11, 395-412 (1997)

35. Srinil, N.: Multi-mode interactions in vortex-induced vibrations of flexible curved/straight structures with geometric nonlinearities. J. Fluids Struct. 26, 1098-1122 (2010)

36. Srinil, N.: Analysis and prediction of vortex-induced vibrations of variable-tension vertical risers in linearly sheared currents. Appl. Ocean Res. 33, 41-53 (2011) 
37. Srinil, N., Zanganeh, H.: Modelling of coupled cross-flow/in-line vortex-induced vibrations using double Duffing and van der Pol oscillators. Ocean Eng. 53, 83-97 (2012)

38. Srinil, N., Zanganeh, H., Day, A.: Two-degree-of-freedom VIV of circular cylinder with variable natural frequency ratio: experimental and numerical investigations. Ocean Eng. 73, 179-194 (2013)

39. Williamson, C.H.K., Govardhan, R.: Vortex-induced vibrations. Annu. Rev. Fluid Mech. 36, 413-455 (2004)

40. Wilson, J., Tinsley, J.: Vortex load reduction: experiments in optimal helical strake geometry for rigid cylinders. J. Energy Res. Technol. 111, 72-76 (1989)

41. Zanganeh, H., Srinil, N.: Characterization of variable hydrodynamic coefficients and maximum responses in two-dimensional vortex-induced vibrations with dual resonances. J. Vib. Acoust. 136, 051010 (2014)

42. Zanganeh, H., Srinil, N.: Three-dimensional VIV prediction model for a long flexible cylinder with axial dynamics and mean drag magnifications. J. Fluids Struct. 66, 127-146 (2016) 